\title{
High resolution phase space measurements with Allison-type emittance scanners
}

\author{
Jonathan C. Wong* \\ National Superconducting Cyclotron Laboratory, Michigan State University, \\ East Lansing, Michigan 48824, USA \\ Steven M. Lund and Tomofumi Maruta \\ Facility for Rare Isotope Beams, Michigan State University, East Lansing, Michigan 48824, USA
}

(Received 29 November 2018; published 2 July 2019)

\begin{abstract}
Allison-type emittance scanners are widely used to measure projected 2D phase space distributions of low energy beams. This paper extends the conventional data analysis model to introduce three significant corrections that commonly arise in the pursuit of high resolution measurements. First, effective longitudinal asymmetry in the E-dipole placement (typically resulting from directional choice of relief cuts in thick slitplates) causes deviation from the ideal voltage-to-angle conversion relation. Second, finite slit thickness generates variation in weights of data points that should be compensated. Third, when the interval between data points is smaller than the device resolution (ordinary in the angular data accumulation), a detailed account of the phase space region contributing to each data point can be used to resolve the beam distribution more accurately. These findings are illustrated by simulations with numerically generated phase space distributions. The improved model is applied to experimental measurements of an Ar ion beam with an Allison scanner operating at the front-end of the Facility for Rare Isotope Beams (FRIB) at Michigan State University. Results show that the improved model obtains better agreement among a set of measurements and modifies beam moments significantly (can be $\sim 10 \%$ relative to conventional methods, with larger deviations at increasing angular divergence), thus rendering the corrections important for accurate high resolution phase-space characterizations. PYTHON code tools that implement the improved analysis described are made available. These tools are readily applicable to any Allison scanner given a specification of the device geometry and scan ranges associated with each measurement.
\end{abstract}

DOI: $10.1103 /$ PhysRevAccelBeams.22.072801

\section{INTRODUCTION}

Allison-type emittance scanners [1], or Allison scanners for short, are widely used to efficiently measure projected 2D phase space distributions of low-energy beams. An Allison scanner (see Fig. 1) consists of an entrance slitplate (slit width $s$ ), an aligned exit slit-plate (slit width $s$ ) with an integrated Faraday cup, and a bipolar-biased electric dipole (voltage $\pm V_{0}$ ) placed between the two slits. The scanner assembly is translated mechanically (typically in discrete steps with a stepper motor) to change the slit position, and the dipole voltage $V_{0}$ is swept at each step to select transmittable angles by varying the bending strength. The scanner samples one grid point in phase space for each coordinate and "E-dipole" voltage setting, with the beam

*cyjwonghk@gmail.com

Published by the American Physical Society under the terms of the Creative Commons Attribution 4.0 International license. Further distribution of this work must maintain attribution to the author(s) and the published article's title, journal citation, and DOI. density taken to be proportional to the current collected by the Faraday cup. The entire projected distribution is measured by recording currents collected over a range of position and voltage values that samples the full phasespace projection.

Analysis of Allison scanner data requires a voltage-toangle conversion and the angular resolution of the device. Idealized relations were derived in Ref. [1] and summarized in Ref. [2]. They constitute the conventional analysis model which assumes the scanner has an idealized geometry with uniform hard-edge dipole fields, thin slit-plates, and longitudinally symmetric placement of the E-dipole.

Extending upon the work in [3], Sec. II augments the conventional analysis model to account for two commonly occurring geometric features that can lead to significant corrections due to: (1) the effective longitudinal asymmetry in E-dipole placement between the slits, and (2) the finite slit-plate thickness. Their effects on particle transmission are studied using both simulations and analytic expressions which show excellent agreement. Asymmetry in E-dipole placement alters the slope of the linear relation between dipole voltage and particle angle. Finite slit thickness 
causes the weight of data points to vary as a decreasing function of their voltage values. The reduction becomes significant at large voltages and when the slits' thicknessto-width ratio $\gtrsim 1$.

When the interval between data points is smaller than the device resolution, the current measured at each data point is a weighted sum of the actual current densities at the data point and its neighbors. Section III shows that a detailed accounting of the phase space region contributing to each data point allows one to deconvolve the effective blur induced by overlapping data points and resolve the beam distribution more accurately. Reconstruction procedures are illustrated and verified using numerically generated phase-space distributions so that improvements are clearly characterized.

Section IV applies the improved model to experimental data from the Allison scanner used in the front-end of the Facility for Rare Isotope Beams (FRIB) at Michigan State University. Raw measurement data first undergo a noise removal scheme, then the processed data are analyzed with both the conventional model and the improved model. Comparisons reveal that the improved model produces more consistent results among a series of scans with changing focusing upstream. Beam moments are modified significantly relative to conventional treatments, with differences growing with the angular divergence of the beam distribution.

The PYTHON code tools used to perform the data analysis and noise removal are described and made available [4]. The modular programs are readily adaptable to other Allison scanners via a change of geometric input parameters.

Section V concludes this study with an outline of directions for future improvements.

\section{ADDITIONAL GEOMETRIC FEATURES}

This section first describes how asymmetric E-dipole placement and finite slit thickness can be modeled in Allison scanners. Effects induced are investigated using simulations that probe particle transmission given the device geometry and E-dipole voltage. The simulation results are accurately modeled by analytic expressions for typical choices of scanner parameters. The implications for data analysis are demonstrated through simulated measurements of ideal particle distributions chosen to highlight those effects.

\section{A. Geometric model}

With reference to a realistic Allison scanner (see Fig. 1), the geometric information relevant to particle transmission and measurement data analysis can be captured using a model with six parameters. A schematic of the model for measuring the $x-x^{\prime}$ phase space is illustrated in Case IV of Fig. 2 where (i) $L$ is the E-dipole plate length; (ii) $l_{1}$ is the distance between the entrance-slit and the E-dipole plate; (iii) $l_{2}$ is the distance between the exit-slit and the E-dipole

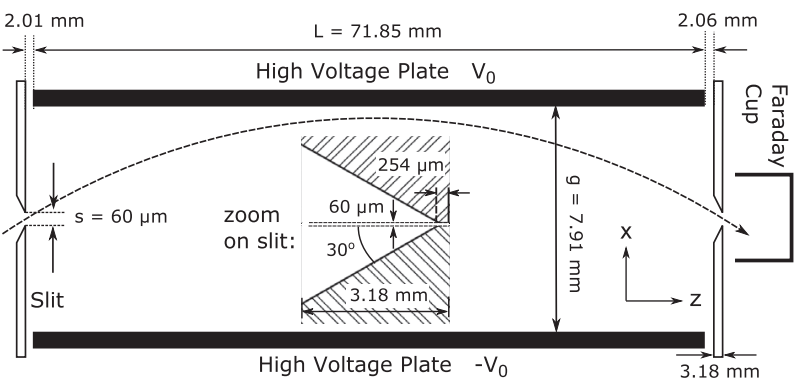

FIG. 1. Side-view of the FRIB Allison scanner.

plate; (iv) $g$ is the gap between the E-dipole plates; (v) $s$ is the slit width; and (vi) $d$ is the slit thickness. The model assumes that particle transmission has no $y$-dependence, which is a good approximation if no particle approaches the vertical extent of the device. This requires the vertical extent of the scanner to be much larger than $2\left(\left|y_{\text {cen }}\right|+y_{\text {rms }}\right)$ where $y_{\text {cen }}$ and $y_{\text {rms }}$ are the beam centroid position and rms width in the vertical direction.

The geometric model hinges upon a correct definition of $l_{1}, l_{2}$, and $d$, so it is important to identify what performs the function of a slit. Figure 1 shows the design for the FRIB Allison scanner [5]. On each side of the E-dipole there is a thick plate with an opening (see zoom insert) that has largeangled relief cuts and a straight channel of minimum width. The relief cuts allow using thicker plates that enable cooling and have higher mechanical stability. The relief cuts do not intercept any ions that would have otherwise passed through the slits, since the opening angle $\left(30^{\circ}\right.$ in Fig. 1) far exceeds the transverse angles of beam ions. Hence the slit, i.e., the structure that limits particle transmission, is only taken to be the minimum-width channel, with width $s=60 \mu \mathrm{m}$ and thickness $d=254 \mu \mathrm{m}$ in Fig. 1 .

The relief cut is immaterial to the slit-to-E-dipole distance $l_{1}$ in Fig. 1 , hence $l_{1}=2.01 \mathrm{~mm}$. In contrast, $l_{2}$, the distance between the dipole plate and the exit slit, must take into account the longitudinal extent of the relief cut because it opens towards the E-dipole. Therefore, $l_{2}=(2.06+3.18-0.254) \mathrm{mm}=4.986 \mathrm{~mm}$. Note that the relief cuts as illustrated in a real system in Fig. 1 induce significant asymmetry in E-dipole placement with $l_{2}=$ $4.986 \mathrm{~mm}>l_{1}=2.01 \mathrm{~mm}$.

Conventional treatments model an Allison scanner with thin slits and symmetry in E-dipole placement, which corresponds to $d=0$ and $l_{1}=l_{2}$. However, real devices often deviate from these assumptions as discussed below.

\section{Longitudinal asymmetry in E-dipole placement $\left(l_{1} \neq l_{2}\right)$}

Effective longitudinal asymmetry in E-dipole placement commonly arises in Allison scanners, probably unintentionally, because relief cuts on thick entrance- and exit-plates have been made in the same direction [6,7]. This asymmetry was also present in the early implementation of the FRIB 
Allison scanner where relief cuts on both plates face the incoming beam as shown in Fig. 1. Since the relief cuts have no effect on particle transmission, it effectively displaces the end slit but not the entrance slit to make $l_{2}>l_{1}$ in the FRIB design. We find that such asymmetries affect the transmitted angles for a given $V_{0}$.

\section{Finite slit thickness $d \gtrsim$ slit width $s$}

The slit thickness $d$ can be neglected when it is much smaller than the slit width $s$. This idealization starts to break down as Allison scanner designs employ decreasing slit widths to improve resolution. Recent examples include slit widths of $s \leq 100 \mu \mathrm{m}$ in plans presented for GSI FAIR [8] and $s=38 \mu \mathrm{m}$ implemented at TRIUMF [9]. Figure 1 details the slit plate presently employed in the FRIB scanner where $s=60 \mu \mathrm{m}$ and the slit thickness (not including irrelevant longitudinal extent of the $30^{\circ}$ relief cut) is $254 \mu \mathrm{m}$. This approximately $4: 1$ aspect ratio effectively produces a narrow channel, which can scrape particles that would have otherwise passed through a slitplate with no thickness.

\section{B. Four cases}

To investigate the effects of asymmetry in dipole placement and finite slit thickness, four Allison scanner models (see Fig. 2) are specified using the parameters listed in Table I. The parameters in Case IV correspond to the geometric model of the FRIB Allison scanner in Fig. 1, which has asymmetric E-dipole placement $\left(l_{1} \neq l_{2}\right)$ and thick slits $(d \neq 0)$. To mimic how the analysis would have been done if one or both geometric features are omitted, the FRIB design is modified into examples of Cases I to III (see Table I) with thin slits $(d=0)$ and/or symmetric E-dipole placement $\left(l_{1}=l_{2}\right)$. Note that Case I corresponds to the conventional analysis model. All four cases have identical E-dipole length $L$, gap $g$ and total inter-slit distance $L+l_{1}+l_{2}$; thus all differences in analysis results arise from the geometric features.

Characteristic trajectories in Fig. 2 illustrate the range of angles that can be transmitted at each voltage setting due to the finite slit width. We define: (i) $x_{\text {ref }}^{\prime}$ : angle at which an ion would enter and exit the slits at the same $x$-position [10], (ii) $x_{\max / \min }^{\prime}$ : maximum/minimum transmittable angle, (iii) $\Delta x^{\prime}=x_{\max }^{\prime}-x_{\min }^{\prime}$ : angular resolution, where $x^{\prime} \equiv$ $d x / d z$ and $x=0$ corresponds to the device center-line. The $x_{\max }^{\prime}$-trajectory touches the lower edge of the entrance slit and the upper edge of the exit slit, and vice versa for the
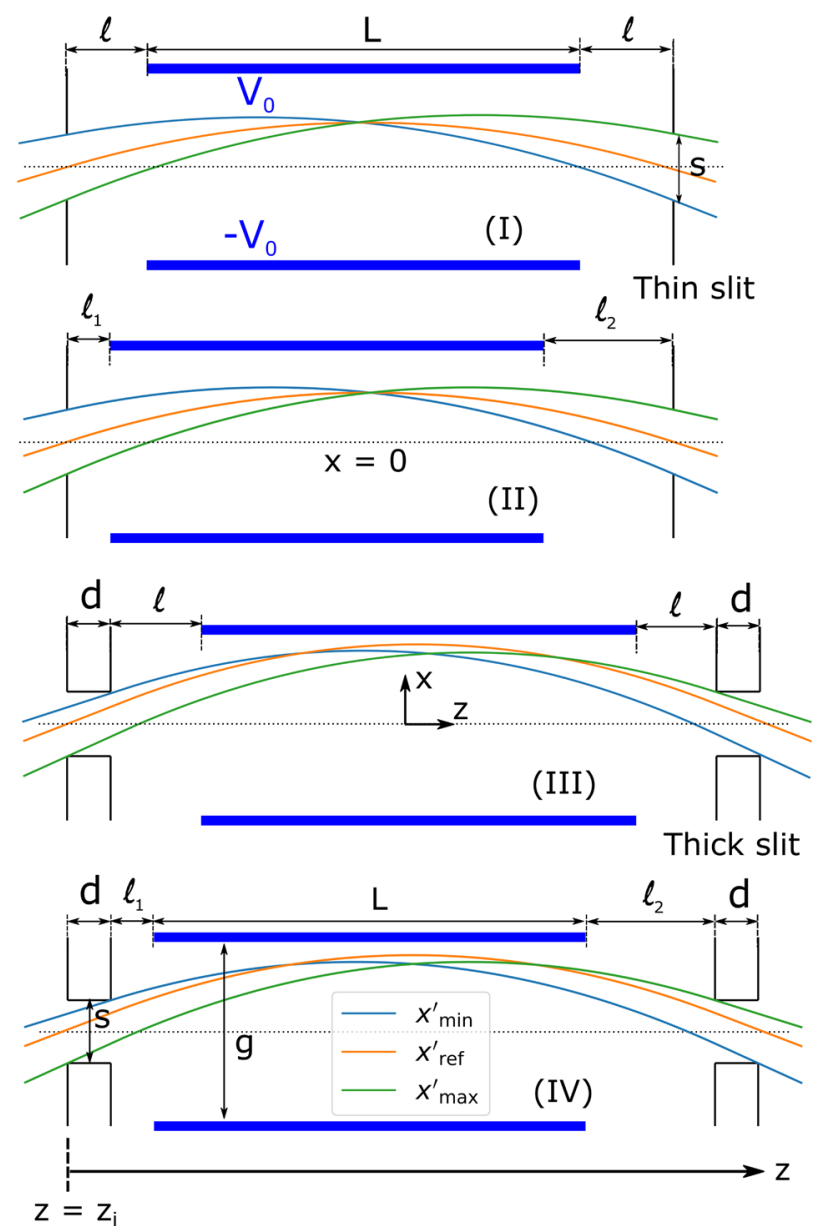

FIG. 2. Four geometric cases and their respective characteristic trajectories. Case I: $l_{1}=l_{2}=l$ and $d=0$; Case II: $l_{1} \neq l_{2}$ and $d=0$; Case III: $l_{1}=l_{2}=l$ and $d \neq 0$; Case IV: $l_{1} \neq l_{2}$ and $d \neq 0 . z_{i}$ is the longitudinal position where the beam particles enter the device and where the $x-x^{\prime}$ phase space is measured.

$x_{\min }^{\prime}$-trajectory. When the slits have finite thickness, one has to determine whether the extreme trajectory should touch the corner on the upstream or downstream side of each slit by selecting the combination that is not scraped. This complicates analytic modeling and results in a longitudinal shift of the extreme trajectories as visualized in Case III and IV of Fig. 2.

\section{Particle simulations}

Transmission properties of an Allison scanner design can be investigated using particle simulations. Two types

TABLE I. Geometric parameters applied in examples corresponding to the four geometric models in Fig. 2.

\begin{tabular}{lcccccccc}
\hline \hline Case & $L[\mathrm{~mm}]$ & $l_{1}[\mathrm{~mm}]$ & $l_{2}[\mathrm{~mm}]$ & $g[\mathrm{~mm}]$ & $s[\mu \mathrm{m}]$ & $d[\mu \mathrm{m}]$ & E-dipole placement & Slits \\
\hline I & 71.85 & 3.498 & 3.498 & 7.91 & 60 & 0 & Symmetric & Thin \\
II & 71.85 & 2.01 & 4.986 & 7.91 & 60 & 0 & Asymmetric & Thin \\
III & 71.85 & 3.498 & 3.498 & 7.91 & 60 & 254 & Symmetric & Thick \\
IV & 71.85 & 2.01 & 4.986 & 7.91 & 60 & 254 & Asymmetric & Thick \\
\hline \hline
\end{tabular}



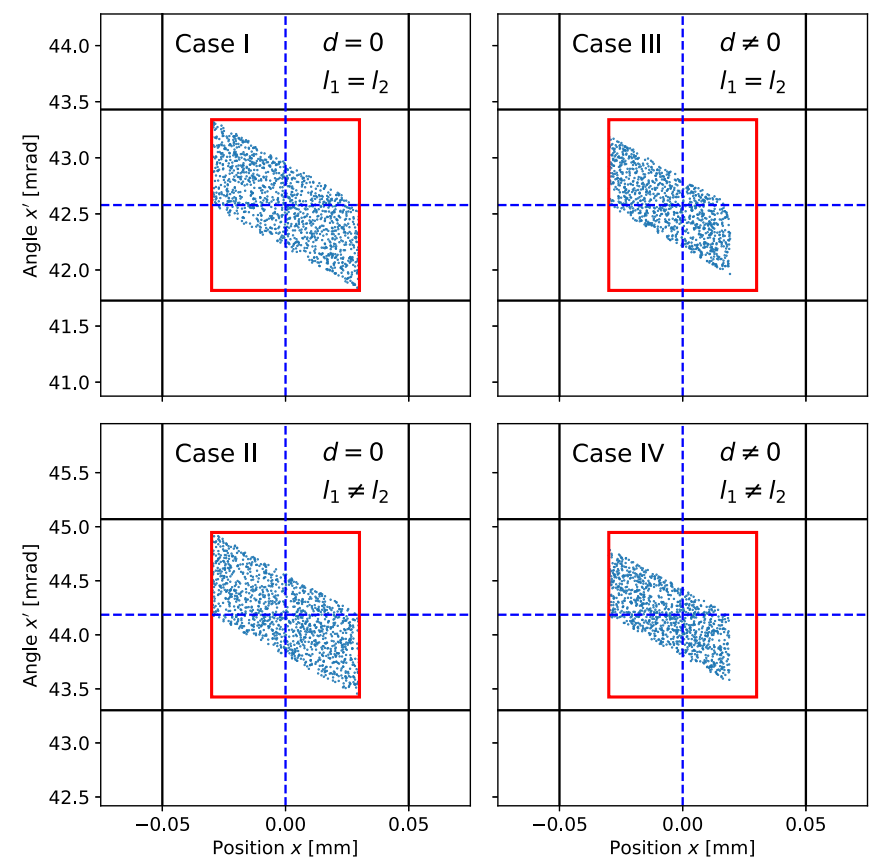

(a) $V_{0}=500 \mathrm{~V}$
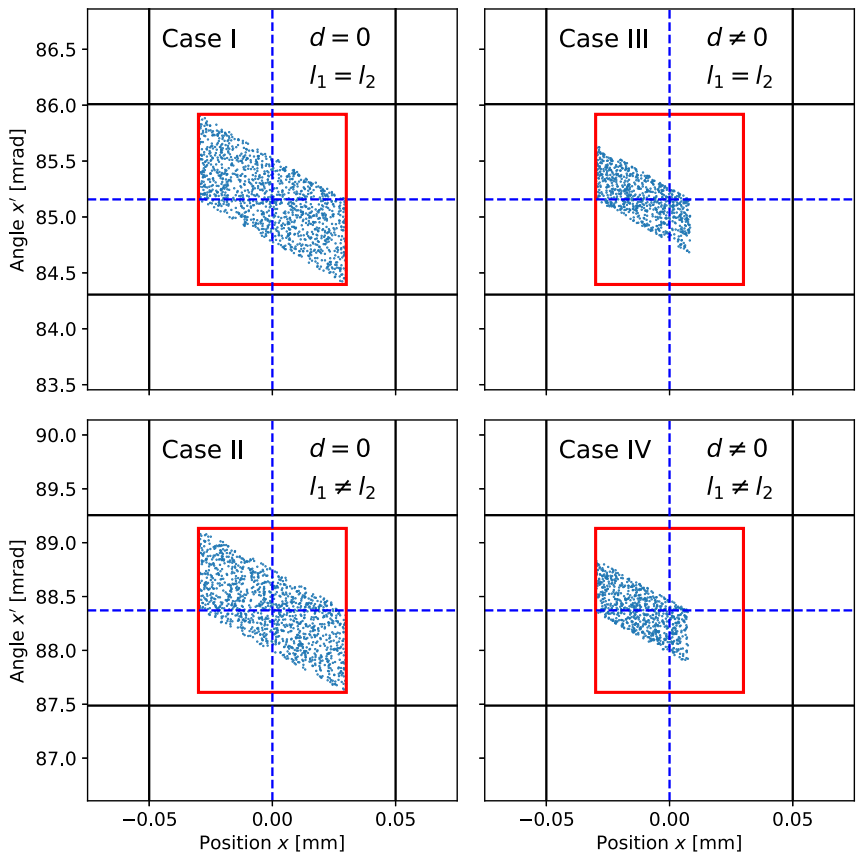

(b) $V_{0}=1000 \mathrm{~V}$

FIG. 3. Blue dots denote particles that are transmitted in the simulations described in Sec. II B 1. They map $x$ - $x^{\prime}$ phase space regions at the device entrance $z=z_{i}$ that contribute to the data point at $x=0 \mathrm{~mm}$. Results are shown for all four geometric cases in Fig. 2 for (a) $V_{0}=500 \mathrm{~V}$ and (b) $V_{0}=1000 \mathrm{~V}$ respectively. Geometric parameters for each case are listed in Table I. Black lines define the phase space grid with increments determined by the scanning step sizes. Dashed blue lines indicate the nominal position $x=0$ and angle $x^{\prime}=x_{\text {ref }}^{\prime}$ corresponding to the data point. The red rectangles denote the device resolution predicted by the conventional treatment (i.e., Case I).

of simulations are employed, namely "ballistic" and "realistic," depending on the model of the dipole field. Realistic simulations employ a numerically calculated fringe field model for the dipole and include nonparaxial effects due to energy change. Ballistic simulations take the Allison scanner as an ideal dipole with uniform hardedge fields over the axial extent $L$, with free drifts and collimating apertures on either side. Energy change from crossing E-dipole potential lines is neglected. Given $V_{0}$, the particle trajectory consists of two straight lines outside the dipole and a parabola inside, and is scraped if it hits either of the slit plates. Space charge and scattering effects are neglected in this study. A condition is derived in Appendix A to show that nonparaxial effects are typically tiny. Details of the realistic model are given in Appendix B.

TABLE II. Analytic formulas corresponding to the geometric models in Fig. 2 for $V_{0} \geq 0$. Results for $V_{0}<0$ obey the same formulas with $V_{0} \rightarrow\left|V_{0}\right|$ and the $x$-axis reversed.

\begin{tabular}{|c|c|c|c|c|}
\hline $\begin{array}{l}\text { Case } \\
\text { Dipole placement } \\
\text { Slit thickness }\end{array}$ & $\begin{array}{c}\mathrm{I} \\
\text { symmetric }\left(l_{1}=l_{2}\right) \\
\text { thin }(d=0)\end{array}$ & $\begin{array}{c}\mathrm{II} \\
\text { asymmetric }\left(l_{1} \neq l_{2}\right) \\
\text { thin }(d=0)\end{array}$ & $\begin{array}{c}\text { III } \\
\text { symmetric }\left(l_{1}=l_{2}\right) \\
\text { thick }(d \neq 0)\end{array}$ & $\begin{array}{c}\mathrm{IV} \\
\text { asymmetric }\left(l_{1} \neq l_{2}\right) \\
\text { thick }(d \neq 0)\end{array}$ \\
\hline$x_{\text {ref }}^{\prime}$ & $\frac{1}{2} \frac{q V_{0} L}{g \mathcal{E}}$ & $\frac{1}{2} \frac{q V_{0} L}{g \mathcal{E}}\left(1+\frac{l_{2}-l_{1}}{L+l_{1}+l_{2}}\right)$ & $\frac{1}{2} \frac{q V_{0} L}{g \mathcal{E}}$ & $\frac{1}{2} \frac{q V_{0} L}{g \mathcal{E}}\left(1+\frac{l_{2}-l_{1}}{L+l_{1}+l_{2}}\right)$ \\
\hline$x_{\max }^{\prime}-x_{\text {ref }}^{\prime}$ & $\frac{s}{L+2 l}$ & $\frac{s}{L+l_{1}+l_{2}}$ & $\frac{s-x_{\mathrm{ref}}^{\prime} d}{L+2 l+d}$ & $\frac{s-x_{\text {ref }}^{\prime} d}{L+l_{1}+l_{2}+d}$ \\
\hline$x_{\mathrm{ref}}^{\prime}-x_{\min }^{\prime}$ & $\frac{s}{L+2 l}$ & $\frac{s}{L+l_{1}+l_{2}}$ & $\frac{s-x_{\mathrm{ref}}^{\prime} d}{L+2 l+d}$ & $\frac{1}{L+l_{1}+l_{2}+d}\left(s-\frac{L+2 l_{1}}{L+2 l_{2}} x_{\mathrm{ref}}^{\prime} d\right)$ \\
\hline$\Delta x^{\prime}$ & $\frac{2 s}{L+2 l}$ & $\frac{2 s}{L+l_{1}+l_{2}}$ & $\frac{2 s}{L+2 l+d}\left(1-\frac{x_{\mathrm{ref}}^{\prime} d}{s}\right)$ & $\frac{2 s}{L+l_{1}+l_{2}+d}\left(1-\frac{L+l_{1}+l_{2}}{L+2 l_{2}} \frac{x_{\mathrm{ref}}^{\prime} d}{s}\right)$ \\
\hline$T\left(x^{\prime}\right)$ for $x^{\prime} \geq x_{\mathrm{ref}}^{\prime}$ & $\frac{x_{\max }^{\prime}-x^{\prime}}{x_{\max }^{\prime}-x_{\text {ref }}^{\prime}}$ & $\frac{x_{\max }^{\prime}-x^{\prime}}{x_{\max }^{\prime}-x_{\mathrm{ref}}^{\prime}}$ & $\frac{x_{\max }^{\prime}-x^{\prime}}{x_{\max }^{\prime}-x_{\mathrm{ref}}^{\prime}}\left(1-\frac{x_{\mathrm{ref}}^{\prime} d}{s}\right)$ & see Appendix C 2 \\
\hline$T\left(x^{\prime}\right)$ for $x^{\prime}<x_{\mathrm{ref}}^{\prime}$ & $\frac{x^{\prime}-x_{\min }^{\prime}}{x_{\mathrm{ref}}^{\prime}-x_{\min }^{\prime}}$ & $\frac{x^{\prime}-x_{\min }^{\prime}}{x_{\mathrm{ref}}^{\prime}-x_{\min }^{\prime}}$ & $\frac{x^{\prime}-x_{\min }^{\prime}}{x_{\mathrm{ref}}^{\prime}-x_{\min }^{\prime}}\left(1-\frac{x_{\mathrm{ref}}^{\prime} d}{s}\right)$ & see Appendix C 2 \\
\hline$W$ & $\frac{1}{2}$ & $\frac{1}{2}$ & $\frac{1}{2}\left(1-\frac{x_{\mathrm{ref}}^{\prime} d}{s}\right)^{2}$ & see Appendix C 2 \\
\hline
\end{tabular}


Most simulation results shown are obtained using ballistic simulations because they are much faster than realistic simulations and can be summarized using analytic formulas. Results in Sec. IIC show that the differences between ballistic and realistic simulations are small for typical scanner geometries.

The device center-line is chosen as the $x$-axis of the coordinate system for simplicity of description. To analyze particle transmission at a given $V_{0}$ value, the incoming angle which sends a particle to the same position at both slits, i.e., $x_{\text {ref }}^{\prime}$, is first obtained numerically via root-finding. Then, assuming that the phase space distribution is uniform over the acceptance region, an ensemble of incident particles are generated under the following conditions. Transversely, the particles have a uniform distribution about $x^{\prime}=x_{\mathrm{ref}}^{\prime}$ and $x=0$ with sufficient widths to fully populate the acceptance. Longitudinally, all particles have the same initial position $z_{i}$ and lie on the plane where the particles enter the device (see Fig. 2). For slits with finite thickness, the particles are aligned with the outer plane of the entrance slit. Every particle is advanced with ballistic simulations and all that can pass through the exit slit are recorded.

Using ${ }^{40} \mathrm{Ar}^{9+}$ ions with kinetic energy $\mathcal{E}=12 \mathrm{keV} / \mathrm{u}$ as test particles, the phase space region that can be transmitted at $V_{0}=500 \mathrm{~V}$ and $V_{0}=1000 \mathrm{~V}$ for the four geometric cases in Table I are plotted in Fig. 3 for ballistic simulations. The red rectangle represents the position resolution $\Delta x_{\text {ideal }}$ and angle resolution $\Delta x_{\text {ideal }}^{\prime}$ predicted by the conventional treatment $([1,2]$, also reproduced under Case I in Table II), where

$$
\begin{aligned}
& \Delta x_{\text {ideal }}=s, \\
& \Delta x_{\text {ideal }}^{\prime}=\frac{2 s}{L+2 l} .
\end{aligned}
$$

A comparison of Cases I and III $\left(l_{1}=l_{2}\right)$ against Cases II and IV $\left(l_{1} \neq l_{2}\right)$ reveals that $x_{\text {ref }}^{\prime}$ changes as a result of longitudinal asymmetry, even though the parameters are chosen such that all cases have the same interslit distance.

The effects of finite slit thickness are manifested in Cases III and IV where the phase space area transmitted shrinks in both dimensions because $d \approx 4 s \neq 0$. Furthermore, the shrinkage is larger in Fig. 3(a) than in Fig. 3(b) - this indicates that this effect increases with $V_{0}$, which corresponds to a larger selected reference angle $x_{\text {ref }}^{\prime}$.

\section{Particle transmission quantified}

To quantify the transmission properties at a specified value of $V_{0}$, assuming the phase space distribution is uniform about $x=0$ and $x^{\prime}=x_{\text {ref }}^{\prime}$, the density of transmitted particles in phase space can be defined as

$$
f\left(x, x^{\prime}\right)=\left\{\begin{array}{ll}
1 & \text { if }\left(x, x^{\prime}\right) \in A \\
0 & \text { if }\left(x, x^{\prime}\right) \notin A
\end{array},\right.
$$

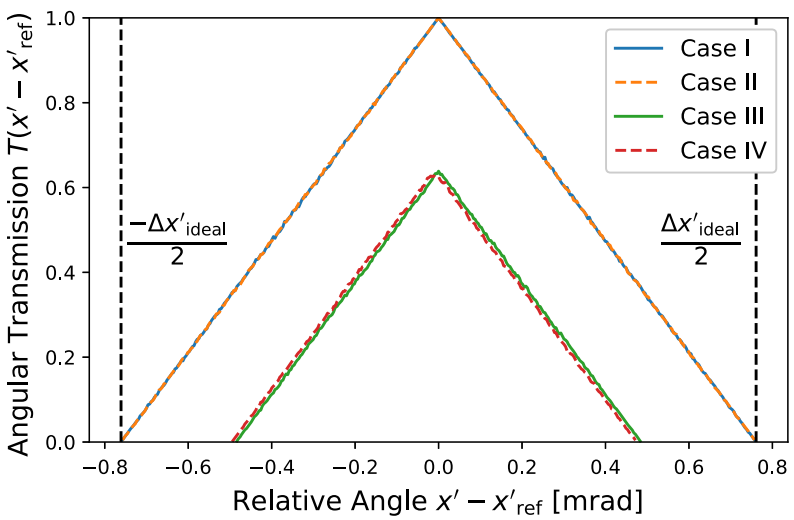

FIG. 4. Angular transmission factor $T\left(x^{\prime}\right)$ for Cases I to IV in Fig. 3(b).

where $A=\left\{\left(x, x^{\prime}\right) \mid\right.$ particle at $\left(x, x^{\prime}\right)$ is transmittable $\}$. Graphically, in Fig. 3, $f\left(x, x^{\prime}\right)=1$ inside the blue area flagging the simulated phase space particles that are transmittable and $f\left(x, x^{\prime}\right)=0$ outside.

Two quantities that distill useful information concerning the transmitted phase space area are the angular transmission factor:

$$
T\left(x^{\prime}\right)=\frac{1}{\Delta x_{\text {ideal }}} \int_{-\Delta x_{\text {ideal }} / 2}^{\Delta x_{\text {ideal }} / 2} f\left(\tilde{x}, x^{\prime}\right) d \tilde{x},
$$

and total transmission factor

$$
W=\frac{1}{\Delta x_{\text {ideal }}^{\prime}} \int_{x_{\text {ref }}^{\prime}-\Delta x_{\text {ideal }}^{\prime} / 2}^{x_{\text {real }}^{\prime}+\Delta x_{\text {ideal }}^{\prime} / 2} T\left(\tilde{x}^{\prime}\right) d \tilde{x}^{\prime} .
$$

Here, $\Delta x^{\prime}$ and $\Delta x_{\text {ideal }}^{\prime}$ are the ideal position and angular resolution of the device described by Eq. (1), and $\tilde{x}$ and $\tilde{x}^{\prime}$ are dummy variables used to denote integration over position and angle respectively.

The angular transmission factor $T\left(x^{\prime}\right)$ represents the fraction of particles entering the slit with angle $x^{\prime}$ that is transmitted, assuming a uniform distribution in initial $x$ over the slit width. $T\left(x^{\prime}\right)$ can be interpreted as a normalized projection of the blue areas in Fig. 3 onto the coordinate $x^{\prime}$ by integrating over $x . T\left(x^{\prime}\right)$ corresponding to the four cases in Fig. 3(b) is plotted in Fig. 4. For Cases I and II with thin slits, $T\left(x^{\prime}\right)=1$ when $x^{\prime}=x_{\text {ref }}^{\prime}$ and decreases linearly until $T\left(x^{\prime}\right)=0$ at $x^{\prime}=x_{\text {ref }}^{\prime} \pm \Delta x_{\text {ideal }}^{\prime}$. Thick slits in Cases III and IV diminish both the range of transmittable $x^{\prime}$ and the fraction of particles transmitted at a given $x^{\prime}$.

The total transmission factor $W$ represents the area ratio between the transmittable phase space region and $\Delta x_{\text {ideal }} \times$ $\Delta x_{\text {ideal }}^{\prime}$ defined by the device resolution. It can be thought of as the ratio between the blue area and area of the red rectangle in any individual plot in Fig. 3. Alternatively, it is the ratio between the area under $T\left(x^{\prime}\right)$ and the area enclosed by the two vertical dotted lines in Fig. 4 . $W$ always satisfies $W \leq 0.5$ with $W=0.5$ corresponding to the ideal geometry 
(Case I). This result means that within the rectangular phase space region defined by the resolution obtained conventionally, no more than half of the area is actually transmittable. The fact that $W$ decreases with $V_{0}$ in Cases III and IV leads to significant corrections in the data analysis.

\section{Analytic results}

Exploiting the simple geometry of particle trajectories in free drifts and ideal dipole fields, all quantities relevant to particle transmission defined in this section can be derived analytically following the steps outlined in Appendix C. Table II summarizes the formulas derived for all four cases. The formulas presented have been numerically verified.

The linear coefficient in the voltage-to-angle relation giving $x_{\text {ref }}^{\prime}$ as a function of $V_{0}$ is shown to change with asymmetry in dipole placement. Expressions for $T\left(x^{\prime}\right)$ in Cases I to III agree with Fig. 4 where $T(x)$ is maximum at $x^{\prime}=x_{\text {ref }}^{\prime}$ and declines linearly to 0 at $x^{\prime}=x_{\max / \min }^{\prime}$. Factors of $\left[1-\left(x_{\text {ref }}^{\prime} d / s\right)\right]$ in Case III arise from effects resulting from the finite slit thickness. The factor is $\leq 1$ and decreases linearly with increasing $V_{0}$ since $x_{\text {ref }}^{\prime} \propto V_{0}$. This effect causes the shrinkage of the blue areas in Fig. 3 when $d \neq 0$.

$T\left(x^{\prime}\right)$ and $W$ in Case IV are more complicated due to the coupling between asymmetric dipole placement and finite slit thickness. Details of the derivation can be found in Appendix C 2. However, in most relevant cases, $T\left(x^{\prime}\right)$ and $W$ of Case III and Case IV are very close except at large angles. This can be observed in Fig. 4 where the two plots almost overlap even at $V_{0}=1000 \mathrm{~V}$ (Fig. 16 in Appendix $\mathrm{C} 2$ highlights the differences when they are discernible). Therefore, the discussion concerning Case III typically applies to Case IV with only small modifications.

The agreement between analytic formulas and simulation results, both ballistic and realistic, is demonstrated in the next section where we discuss how the additional geometric features lead to two corrections.

\section{Two corrections in data analysis}

Analytic and simulation results above reveal two significant corrections on the data analysis of Allison scanners relative to the conventional treatment that assumes symmetric dipole placement and thin slits [1,2]. One correction arises from asymmetry in dipole placement and alters the proportionality constant in the linear relation between dipole voltage $\left(V_{0}\right)$ and selected angle $\left(x_{\text {ref }}^{\prime}\right)$. The other correction adjusts the weights of data points as a function of $x_{\text {ref }}^{\prime}$ to compensate for scraping due to the finite slit thickness. Both corrections can be applied using the quantities analytically expressed in Table II. We illustrate these effects for the FRIB Allison scanner geometry shown in Fig. 1 for an ${ }^{40} \mathrm{Ar}^{9+}$ ion with kinetic energy $\mathcal{E}=12 \mathrm{keV} / \mathrm{u}$.

\section{Voltage-to-angle relation}

As shown in Cases II and IV of Table II, when the dipole placement is asymmetric, the reference angle $x_{\text {ref }}^{\prime}$ has an additional factor $\left[1+\left(l_{2}-l_{1}\right) /\left(L+l_{1}+l_{2}\right)\right]$ relative to the symmetrical model results (Case I and III). For the FRIB Allison scanner, $l_{2}-l_{1}=2.976 \mathrm{~mm}$, so the relief cuts generate substantial effective asymmetry. In Fig. 5, $x_{\text {ref }}^{\prime}$ versus $V_{0}$ is plotted for the symmetrical treatment (Table II, Case I or III), improved analytic results (Table II, Case II or IV), and both ballistic and realistic numerical simulations. Both types of simulations agree almost perfectly with analytic results to show a slope of $88.4 \mathrm{mrad} / \mathrm{kV}$, which deviates from the symmetrical result $85.2 \mathrm{mrad} / \mathrm{kV}$ by roughly $4 \%$ as a consequence of the effective asymmetry. The close correspondence between realistic simulations and analytic results shows that fringe-fields and nonparaxial effects impose minimal changes on particle trajectories for typical device geometries and beam parameters, thus validating the analytic calculations as an accurate and efficient way to obtain $x_{\text {ref }}^{\prime}$.

\section{Voltage-dependent weight compensation}

As mentioned in Sec. II B, thick slits cause $W$ to decrease as a function of $x_{\text {ref }}^{\prime}$. To understand implications of this effect, it is important to delineate what the blue area, red rectangle, and black rectangle represent in Fig. 3.

Phase-space scans are typically performed by measuring the current at regular discrete steps in slit position $(x)$ and voltage $\left(V_{0}\right)$. After voltage-to-angle conversion, the data are represented by a grid in phase-space where each grid represents a rectangle of size (position step) $\times($ angle step). In general, grid dimensions will not coincide with the spatial and angular resolution of the device. In Fig. 3, the black rectangle denotes the phase space grid while the conventional device resolution defines the red rectangle. In this study, scanning steps exceed the respective device resolution in both position and angle, as is typical of scans taken in FRIB (see Sec. IV).

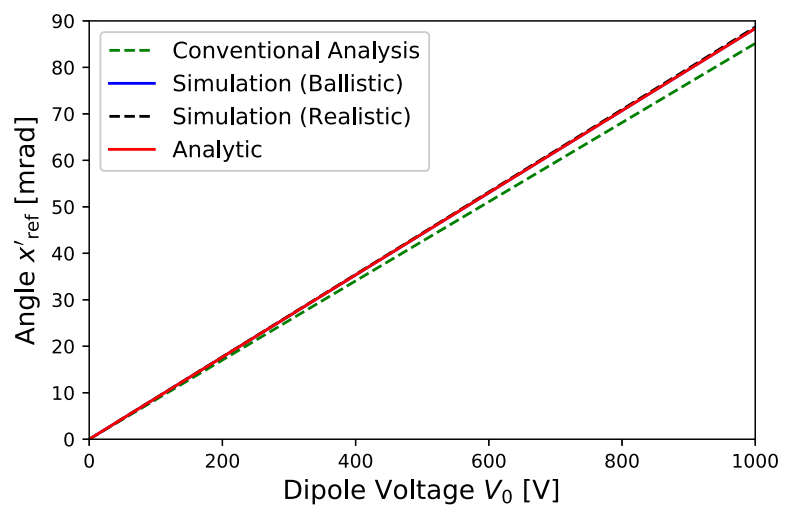

FIG. 5. Voltage-to-angle relation for the selected beam. The red curve (analytic) overlays the blue curve (ballistic simulation). 


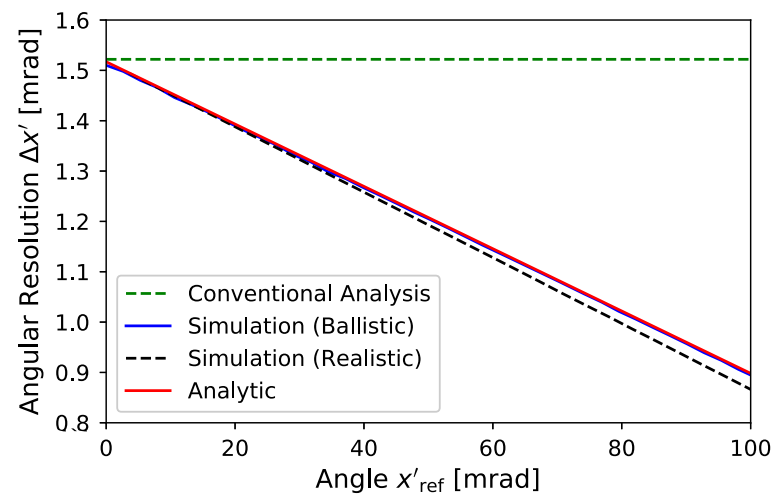

(a)

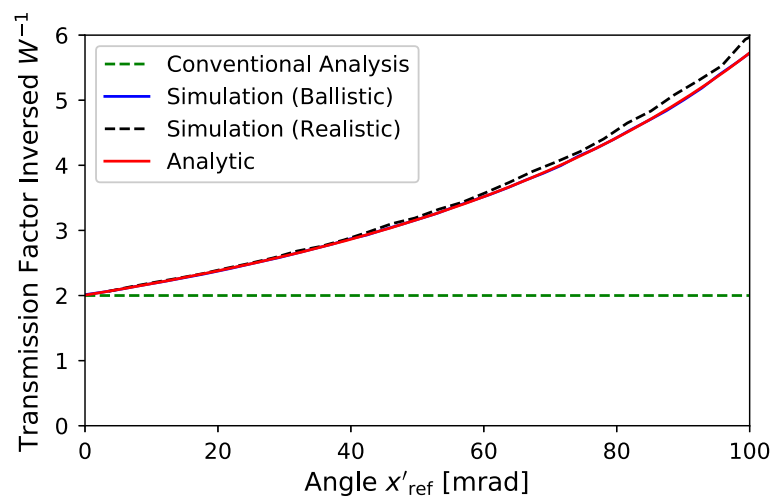

(b)

FIG. 6. Plots of (a) angular resolution $\Delta x^{\prime}$, and (b) angular correction factor as a function of $x_{\text {ref }}^{\prime}$. The red curve (analytic) overlays the blue curve (ballistic simulation).

Assuming a uniform distribution of particles within each grid, the actual beam current falling within each phase space grid cell should be calculated by

$$
I_{\text {grid }}=I_{\mathrm{fc}} \times \frac{\text { Black Rectangle Area }}{\text { Red Rectangle Area }} \times \frac{1}{W},
$$

where $I_{\mathrm{fc}}$ is the Faraday cup current collected at the data point, and $W$ is the ratio between blue area and red rectangular area defined in Sec. II B.

With thin slits, Eq. (5) merely rescales all data points uniformly because $W=1 / 2$ is a constant. Thus one does not need to distinguish between $I_{\text {grid }}$ and $I_{\mathrm{fc}}$ when one calculates moments of the beam distribution in phase space with measured data, unless one also wants to compute the total beam current accurately to verify normalizations.

With thick slits, $W$ is not constant and decreases quadratically in $\left(x_{\mathrm{ref}}^{\prime} \times d / s\right)$ (see Table II). Therefore, $I_{\mathrm{fc}}$ has to be rescaled differently depending on the angle $x_{\text {ref }}^{\prime}$. The larger the slit thickness-to-width ratio $d / s$, the more important this effect becomes. This effect must be taken into account for accurate moment calculations.

In Fig. 6, the angular resolution $\Delta x^{\prime}$ and $1 / W$ are plotted as a function of $x_{\text {ref }}^{\prime}$ for the FRIB Allison scanner. Curves for conventional treatment (Table II, Case I), improved analytic results (Table II, Case II or IV), and numerical simulations are shown. Note again the minimal difference between ballistic and realistic simulation results.

The correction introduced by the effect of finite slit thickness can be substantial. For example, in Fig. 6(b), when $x_{\text {ref }}^{\prime}=70 \mathrm{mrad}, W^{-1} \approx 4$ as opposed to $W^{-1}=2$ when $x_{\text {ref }}^{\prime}=0 \mathrm{mrad}$. Therefore, if we apply the conventional model where all data points have equal weights, 0 -mrad data points would wrongly weigh twice as much as \pm 70 -mrad data points. Failure to correct the weights will distort beam measurements and moment evaluations with increasing amplitude as beam angular extent increases.

In the laboratory, increasing the angular extent of the beam phase space distribution at the Allison scanner by adjusting the applied focusing lattice is often needed to obtain sufficient angular information. An order-of-magnitude inspection based on ideal device resolution in Eq. (1) reveals the reason. $(L+2 l)$ typically $\sim 0.1 \mathrm{~m}$, therefore

$$
\frac{\Delta x_{\text {ideal }}}{\Delta x_{\text {ideal }}^{\prime}} \simeq \frac{L+2 l}{2} \simeq \frac{1}{20} \frac{\mathrm{mm}}{\mathrm{mrad}} .
$$

Since measurements usually take scanning steps exceeding the device resolution, Eq. (6) says that if a similar level of detail is desired in both dimensions, the distribution's angular extent in mrad should be around 20 times its position counterpart in $\mathrm{mm}$. This often forces one to employ relatively narrow scanner slit width $s$ at the expense of reduced Faraday cup currents that lead to greater noise issues, particularly for heavy-ion beams whose currents are typically lower than that of proton beams.

\section{Analysis of synthetic data}

The effects of the two corrections on data analysis can be tested using synthetic measurement data as follows.

After generating an idealized distribution of particles in phase space with given Twiss parameters and emittance, the transmission of each particle at each $\left(x, V_{0}\right)$ pair of the scan can be tested using ballistic simulations. The particle number transmitted at each $\left(x, V_{0}\right)$ setting is recorded to emulate the measurements that would have been taken by an Allison scanner. It is unnecessary to test every $\left(x, V_{0}\right)$ pair for a given particle as many pairs are ruled out based on the device resolution. The artificial data set thus synthesized is processed using both the conventional and improved analysis to analyze errors in beam moments and phase space distortions in comparison with the idealized distribution.

Beam moments can be defined in terms of a continuous particle distribution function representing the beam as:

$$
\left\langle g\left(u, u^{\prime}\right)\right\rangle \equiv \frac{\int_{-\infty}^{\infty} \int_{-\infty}^{\infty} f\left(u, u^{\prime}\right) g\left(u, u^{\prime}\right) d u d u^{\prime}}{\int_{-\infty}^{\infty} \int_{-\infty}^{\infty} f\left(u, u^{\prime}\right) d u d u^{\prime}}
$$



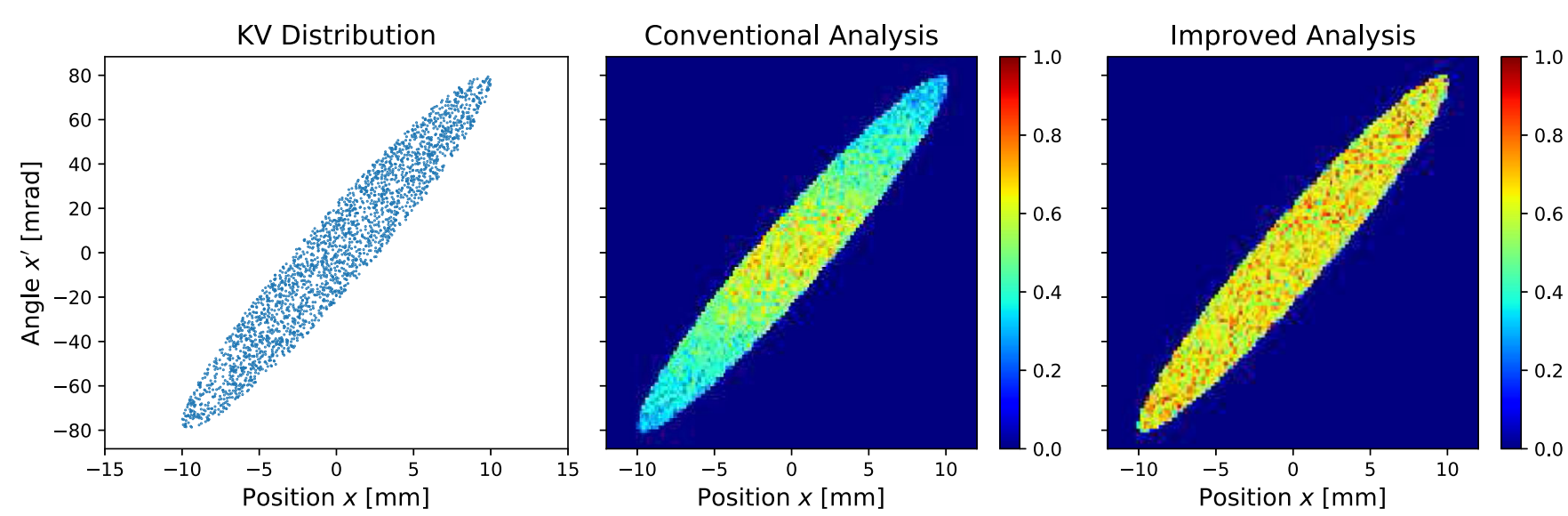

FIG. 7. Uniform phase space projection of the KV distribution, compared against results of synthesized measurement data analyzed using conventional and improved analysis methods. The left plot shows a sampling (1 out of 1000 particles) used to represent the distribution. The middle and right plots are fine mesh density plots with the same color scale.

where $f\left(u, u^{\prime}\right)$ is the distribution function in $u-u^{\prime}$ phase space, and $g\left(u, u^{\prime}\right)=u^{a_{1}} u^{\prime a_{2}}$ with $a_{1}+a_{2}$ being the order of the moment. Here, $u$ represents either $x$ or $y$. Beam moments can be equivalently calculated from the particle perspective as:

$$
\left\langle g\left(u, u^{\prime}\right)\right\rangle=\frac{1}{N} \sum_{i=1}^{N} g\left(u_{i}, u_{i}^{\prime}\right)
$$

for a distribution with $N$ discrete particles, and within a gridded phase space as:

$$
\left\langle g\left(u, u^{\prime}\right)\right\rangle=\frac{\sum_{i=1}^{m} \sum_{j=1}^{n} J_{i j} g\left(u_{j}, u_{i}^{\prime}\right)}{\sum_{i=1}^{m} \sum_{j=1}^{n} J_{i j}}
$$

where $J_{i j}$ is the current density in $u-u^{\prime}$ phase space in the $i, j$ cell. Equation (9) is equivalent to discretizing Eq. (7).

A data set for the Allison scanner geometry in Case IV of Table I is generated using a $12 \mathrm{keV} / \mathrm{u}{ }^{40} \mathrm{Ar}^{9+}$ beam with KV distribution $[11,12]$. The KV distribution has uniform projected density with an $x-x^{\prime}$ phase space ellipse. This simple uniform projection makes it easy to visualize distribution distortions. The KV distribution and results

TABLE III. Beam parameters of the three distributions in Fig. 7.

\begin{tabular}{lccc}
\hline \hline Parameters & $\begin{array}{c}\mathrm{KV} \\
\text { distribution }\end{array}$ & $\begin{array}{c}\text { Conventional } \\
\text { analysis }\end{array}$ & $\begin{array}{c}\text { Improved } \\
\text { analysis }\end{array}$ \\
\hline$\langle x x\rangle\left[\mathrm{mm}^{2}\right]$ & 25.0 & 20.9 & 25.0 \\
$\left\langle x x^{\prime}\right\rangle\left[\mathrm{mm} \mathrm{mrad}^{\prime}\right]$ & 192.0 & 156.5 & 192.3 \\
$\left\langle x^{\prime} x^{\prime}\right\rangle\left[\mathrm{mrad}^{2}\right]$ & 1600 & 1304 & 1603 \\
$\alpha_{x}$ & -3.43 & -3.00 & -3.43 \\
$\beta_{x}[\mathrm{~m}]$ & 0.446 & 0.400 & 0.447 \\
$\varepsilon_{\text {rms }}[\mathrm{mm} \mathrm{mrad}]$ & 56.0 & 52.1 & 56.0 \\
$I_{\text {total }}[\mathrm{mA}]$ & 2.00 & 1.49 & 2.00 \\
\hline \hline
\end{tabular}

from conventional and improved analysis methods are plotted in Fig. 7. Although the KV distribution has a uniform $x-x^{\prime}$ projection within an elliptical boundary by construction, in the synthesized measurement, the beam density artificially diminishes strongly at larger angles under the conventional analysis, whereas the correct uniformity is accurately preserved under the improved analysis.

Beam moments and the associated Twiss parameters $\alpha_{x}$, $\beta_{x}$ with rms emittance $\varepsilon_{\mathrm{rms}}$ are listed in Table III. The results of the improved analysis match the simulated distribution very closely, whereas there is $>10 \%$ deviation in the Twiss parameters if the conventional analysis is used. The conventional analysis also underestimates the total beam current by $25 \%$. These deviations in the conventional analysis will amplify if the angular extent of the KV distribution increases.

\section{CORRECTIONS FOR DEGENERATE PHASE SPACE MEASUREMENTS}

In the previous section, all analysis was made assuming that the measurement step size of the scan always exceeds the device resolution. This assumption is illustrated in Fig. 3, where the red rectangle denoting the device resolution is completely encompassed by the black grid defined by the interval between data points. In this section, we discuss how to correct measurement results when this assumption is no longer valid.

Intuitively, when the device resolution exceeds the grid size, the current measured has contributions from multiple grid cells. Assuming this occurs only in the angle coordinate but not in the position coordinate, Fig. 8 shows an example where the phase space region extends over five grid cells vertically. The current measured at a data point is thus a weighted sum of the current densities from contributing grid cells. Assuming uniform current density in each 

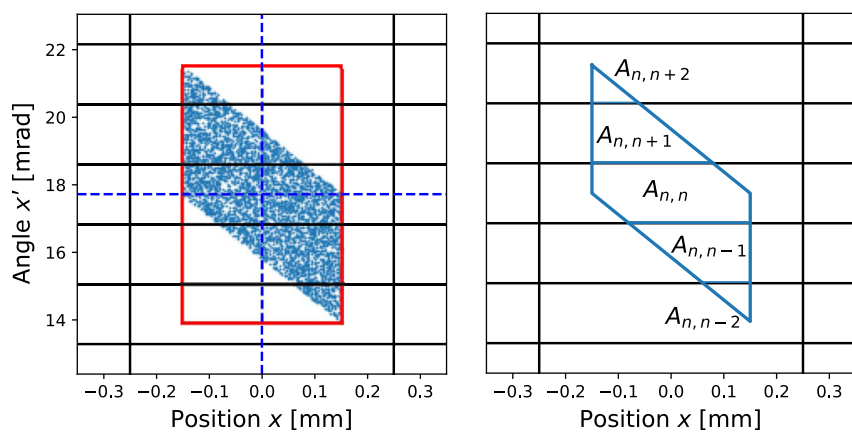

FIG. 8. Phase space region contributing to the data point at $x=0 \mathrm{~mm}$ and $V_{0}=200 \mathrm{~V}$ (presentation format as detailed in Fig. 3), where the Allison scanner geometry corresponds to Case IV of Table I except for increased slit width to $s=300 \mu \mathrm{m}$. Scanning step sizes in $x$ and $V_{0}$ are $0.5 \mathrm{~mm}$ and $20 \mathrm{~V}$ respectively.

grid cell, the weighting factor equals the overlapping area between the blue quadrilateral and the grid cell. Therefore, for each column in the phase space plot, if the array of angular values are numbered from 1 to $n$, a system of linear equations can be written down

$$
\left(\begin{array}{ccc}
A_{1,1} & \cdots & A_{1, n} \\
\vdots & \ddots & \vdots \\
A_{n, 1} & \cdots & A_{n, n}
\end{array}\right)\left(\begin{array}{c}
j_{1} \\
\vdots \\
j_{n}
\end{array}\right)=\left(\begin{array}{c}
I_{1} \\
\vdots \\
I_{n}
\end{array}\right),
$$

where $I_{n}$ is the current measured at the $n$th angle value, $A_{n, m}$ is the area in $m$ th grid occupied by the phase space region contributing to $I_{n}$, and $j_{n}$ is the current density at the $n$th grid. Figure 8 shows several elements of the matrix $\mathbf{A}$, where $I_{n}$ corresponds to the current measured at $V_{0}=200 \mathrm{~V}$ in this case.

Utilizing detailed information from Sec. II on the phase space region contributing to a data point, the matrix $\mathbf{A}$ can be obtained via transmission simulations or numerical integration of $T\left(x^{\prime}-x_{\text {ref }}^{\prime}\right)$ (see Table II). The current densities $j$ can thus be solved for as unknowns to deconvolve the information intermixed in current measurements.

Corrections for degenerate measurements are tested using a synthesized data set generated with the methods described in Sec. II D using a waterbag distribution. The waterbag distribution $[11,12]$ is applied corresponding to uniform transverse energy in phase space up to a sharp
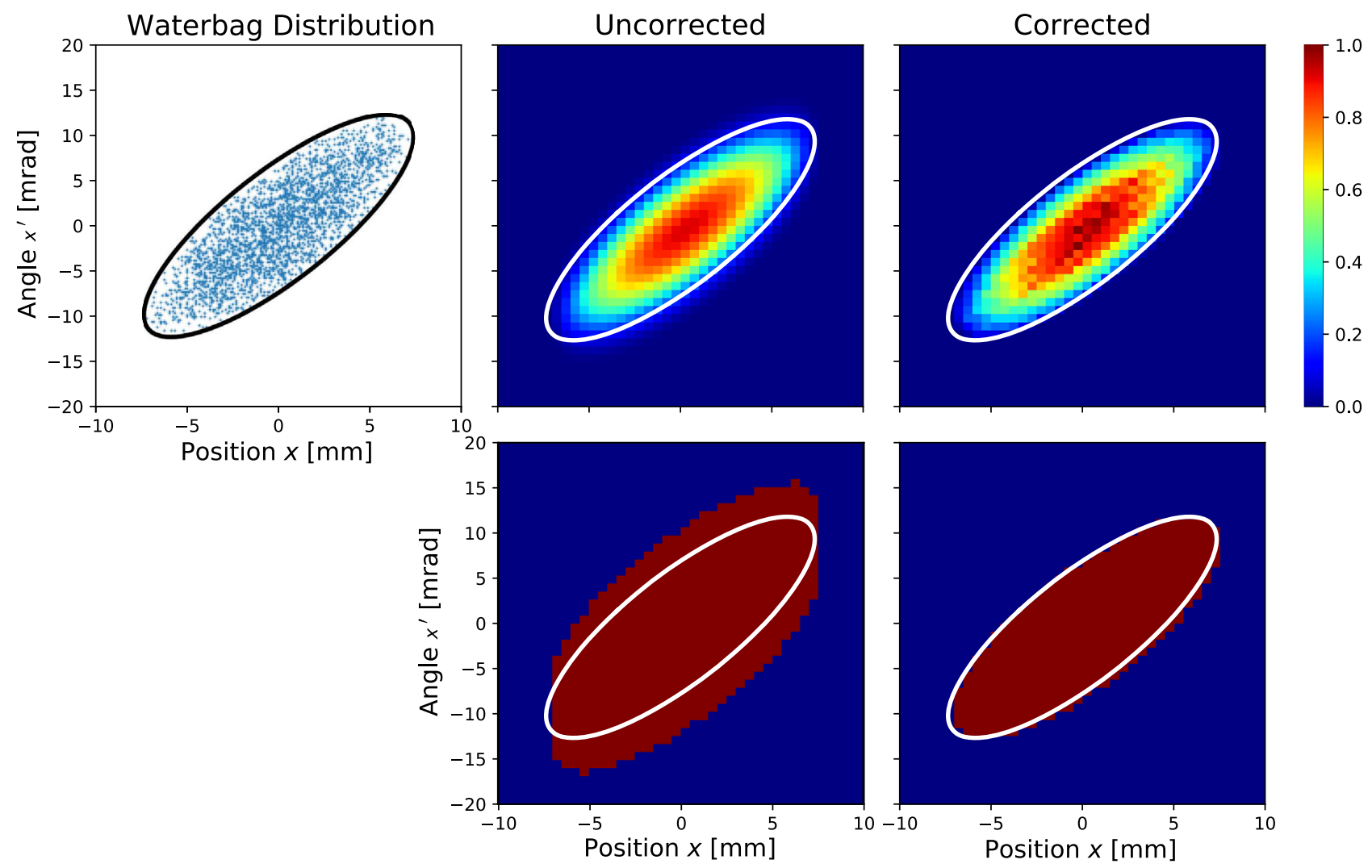

FIG. 9. Waterbag distribution (left column), compared against synthesized measurement data analyzed without (middle column) and with (right column) corrections for degenerate measurements. The upper row corresponds to intensity, whereas the lower row shows all nonzero intensity points in the same color to highlight extent errors. Black and white ellipses denote the ideal boundary of the waterbag distribution analyzed. In the left column sampled particles (1 out of 1000) are plotted. Measurement data are synthesized with $s=300 \mu \mathrm{m}$, and scanning step sizes of $0.5 \mathrm{~mm}$ and $10 \mathrm{~V}$. 
TABLE IV. Beam parameters of the two distribution in Fig. 9 compared against those of the waterbag distribution.

\begin{tabular}{lccc}
\hline \hline Parameters & $\begin{array}{c}\text { Waterbag } \\
\text { distribution }\end{array}$ & $\begin{array}{c}\text { Conventional } \\
\text { analysis }\end{array}$ & $\begin{array}{c}\text { Phase space } \\
\text { correction }\end{array}$ \\
\hline$\langle x x\rangle\left[\mathrm{mm}^{2}\right]$ & 9.00 & 9.03 & 9.03 \\
$\left\langle x x^{\prime}\right\rangle\left[\mathrm{mm} \mathrm{mrad}^{\prime}\right]$ & 12.00 & 11.91 & 11.91 \\
$\left\langle x^{\prime} x^{\prime}\right\rangle\left[\mathrm{mrad}^{2}\right]$ & 25.00 & 27.48 & 25.01 \\
$\alpha_{x}$ & -1.33 & -1.16 & -1.30 \\
$\beta_{x}[\mathrm{~m}]$ & 1.00 & 0.88 & 0.99 \\
$\varepsilon_{\text {rms }}[\mathrm{mm} \mathrm{mrad}]$ & 9.00 & 10.31 & 9.17 \\
$I_{\text {total }}[\mathrm{mA}]$ & 2.00 & 2.00 & 2.00 \\
\hline \hline
\end{tabular}

cutoff value, with self-field energy neglected. The Allison scanner geometry is the same as Case IV of Table I except $s=300 \mu \mathrm{m}$ (i.e., wider slits), while the scanning step size in $V_{0}$ is $10 \mathrm{~V}$. The uncorrected and corrected phase space distributions are plotted in Fig. 9. Beam parameters are listed in Table IV.

Note that the uncorrected phase space distribution appears smoother than the corrected distribution because the uncorrected current measured at each grid point takes contributions from multiple neighboring grid cells, thereby causing a blurring effect. In other words, while the grid size is determined by the scanning step size and can be made arbitrarily small, details finer than the device resolution are misleading unless corrections are applied to deconvolve overlapping measurements.

Figure 9 shows that the uncorrected distribution also has ghost tails that leak out of the $100 \%$ beam ellipse on both sides of the angular extent of the distribution. The effect is illustrated clearly when all nonzero points are colored equally (second row in Fig. 9). This arises because currents measured at points outside the ellipse also take contribution from interior grid points, so the extent of the leakage is determined by the device resolution. Figure 10 shows that such ghost tails can cause significant overestimation of particles with large angles. Therefore, a correct characterization of degenerate measurements and device resolution is potentially important for studies of beam halo where particles outside the core can extend to large angles.

The treatment above assumes the device resolution only exceeds the step size in $x^{\prime}$ but not in $x$. This is quite common because the angular resolution limit is much more likely to be reached by the angular step size, rather than the same occurring in the position dimension, due to the ease of electronically sweeping and recording voltage steps relative to mechanical translation of the scanner (see also last paragraph of Sec. II C). If the step size in $x$ is smaller than $s$, the method above can be extended to $2 \mathrm{D}$ whereby all points in the plot have to be solved at once. This can result in a very large matrix inversion problem unless contributions are limited to some level of nearestneighbor zones.

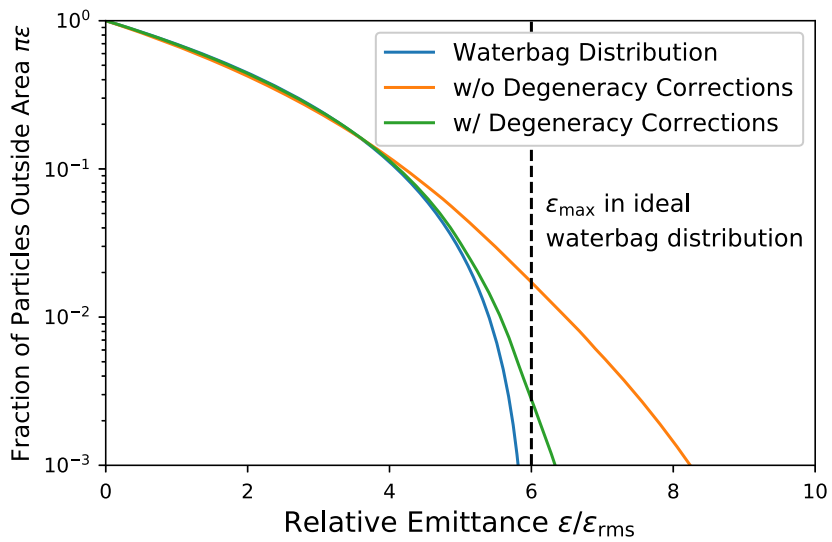

FIG. 10. Fraction of particles that fall outside the ellipse (orientation defined by Twiss parameters) with area $\pi \varepsilon$ in each of the three distributions in Fig. 9. The vertical dashed line corresponds to the sharp phase space edge of the waterbag distribution at $\varepsilon / \varepsilon_{\mathrm{rms}}=6$. The blue curve approaches $\varepsilon / \varepsilon_{\mathrm{rms}}=6$ if the lower range of the ordinate is extended.

\section{EXAMPLE: FRIB ALLISON SCANNER}

This section applies the improved analysis model on measurements at the front end of the Facility for Rare Isotope Beams (FRIB). The FRIB front end is described in Sec. IVA. Section IV B highlights the discrepancies between conventional and improved analysis results, and validates the improved treatment by demonstrating that it delivers more consistent results among a series of measurements performed in a quadrupole scan.

The PYTHON code tools we employ for data analysis of Allison scanner measurements have been made publicly available in a git [13] software repository [4]. Tools include an adaptable code to implement the ideal and improved analysis methods described in Sec. II, and algorithms to process measurement data for noise thresholding. These tools are self-contained, documented, and readily adaptable to a whole variety of applications. They are made available as a community resource. The version used in this study corresponds to November 2018. Future algorithm advances will also be posted and documented in the repository.

\section{A. FRIB front end}

The front end of the FRIB [14] began commissioning in June 2017. A schematic of the upstream end of the low energy beam transport (LEBT) line is shown in Fig. 11. Beam ions are produced in an Artemis-type ECR source [15] with a $15 \mathrm{kV}$ extraction voltage. The beam then traverses a short transport section with solenoid focusing and an electrostatic gap biased to accelerate the target ion species to $12 \mathrm{keV} / \mathrm{u}$. Species are separated horizontally (in $x$ ) via a large dispersion generated in a $90^{\circ}$ magnetic dipole. This is followed by an electrostatic quadrupole 


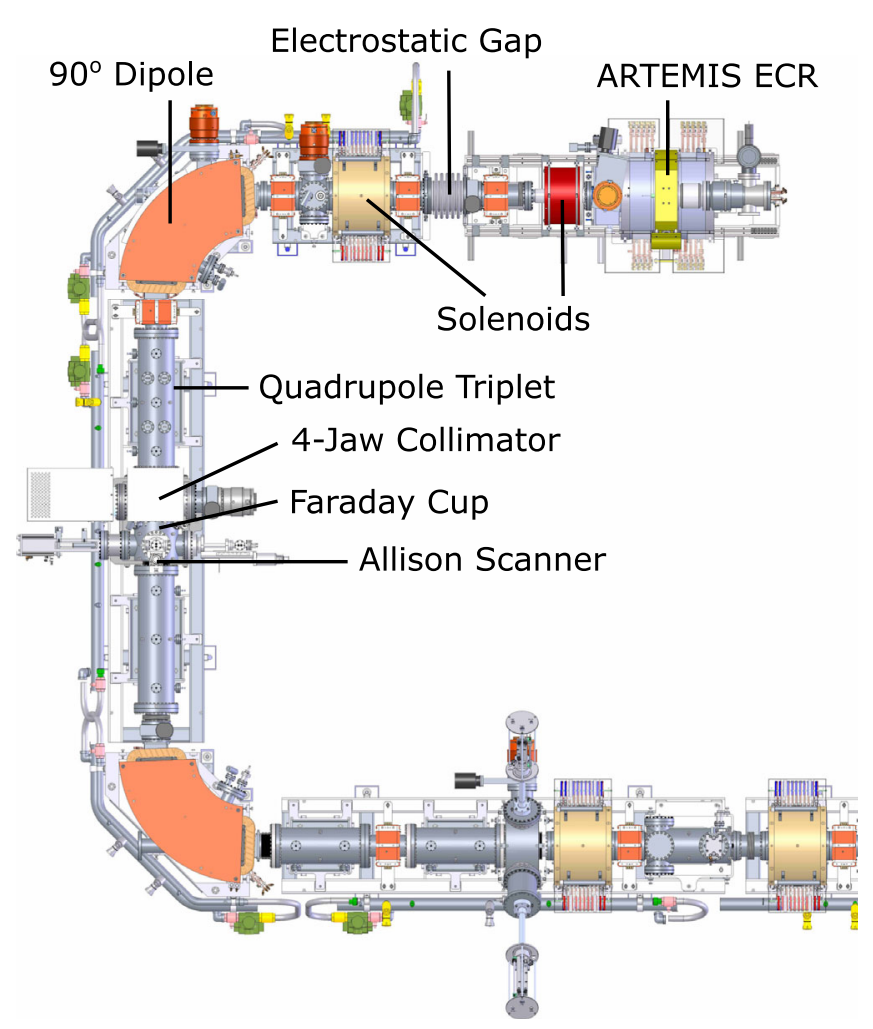

FIG. 11. Upstream end of the low energy beam transport (LEBT) line of the FRIB front end.

triplet and a four-jaw collimator to scrape unwanted ion species in a high dispersion region. Two Allison scanners, one for each transverse direction, are located downstream of the four-jaw collimator. The collimator jaws are adjusted such that only the target species and possibly traces of contaminant ions whose mass-to-charge ratios $M / Q$ differ from the target ion species by $<1 \%$ remain.

The initial implementation of the Allison scanner had geometries corresponding to Case IV in Table I. Motivated by this work, the direction of the relief cuts on the entrance slit plate was flipped in February 2018, thus greatly reducing the longitudinal asymmetry in E-dipole placement. Table V compares the geometries before and after the modification. All measurements in this section were obtained under the new device geometry, hence the corrections have negligible contribution from asymmetry and mainly arise from thick slit effects.

TABLE V. Allison scanner geometries at FRIB front end, before and after modification in Feb 2018.

\begin{tabular}{lcccccc}
\hline \hline & $L$ & $l_{1}$ & $l_{2}$ & $g$ & $s$ & $d$ \\
Design & {$[\mathrm{mm}]$} & {$[\mathrm{mm}]$} & {$[\mathrm{mm}]$} & {$[\mathrm{mm}]$} & {$[\mu \mathrm{m}]$} & {$[\mu \mathrm{m}]$} \\
\hline Before symmetrization & 71.85 & 2.01 & 4.986 & 7.91 & 60 & 254 \\
After symmetrization & 71.85 & 4.938 & 4.986 & 7.91 & 60 & 254 \\
\hline \hline
\end{tabular}
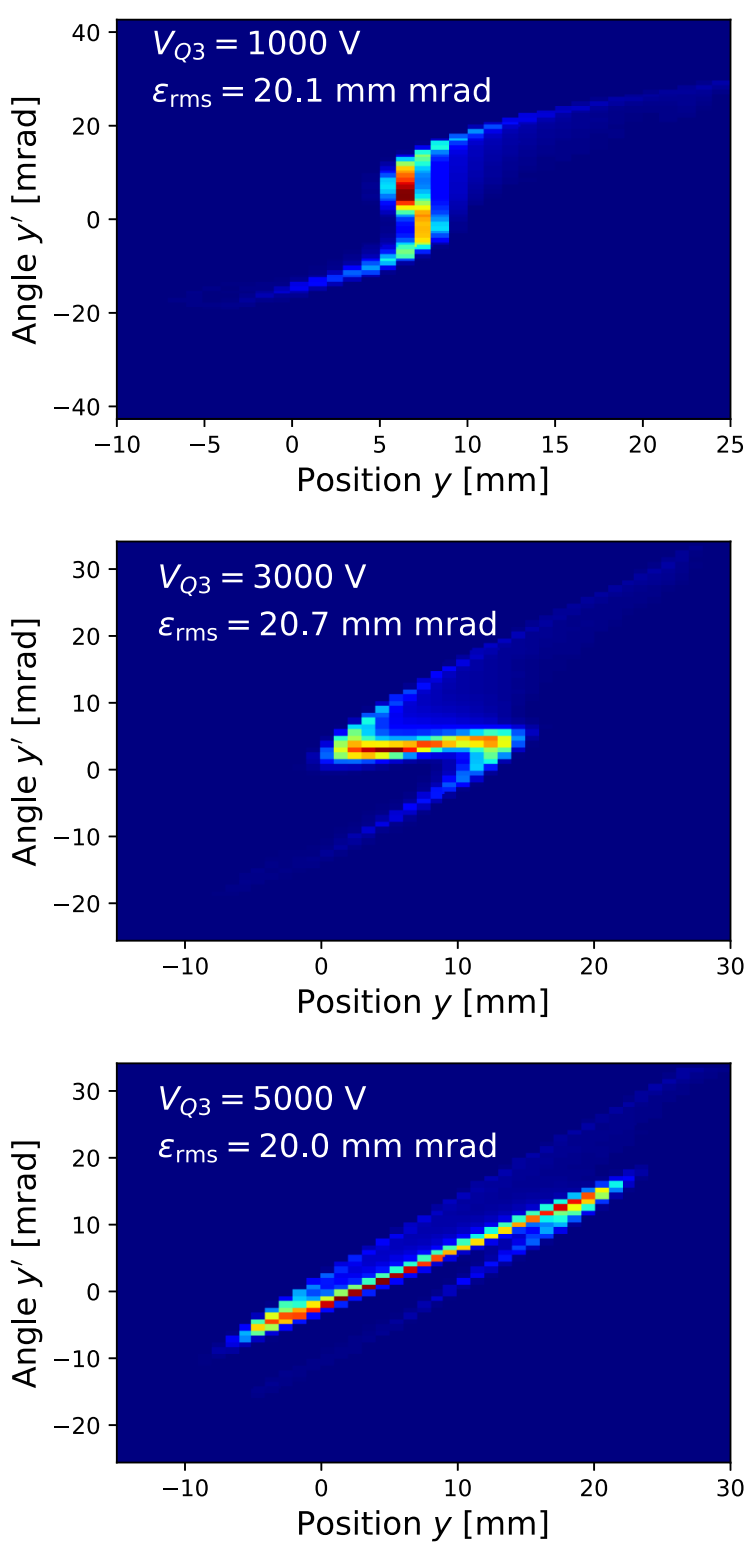

FIG. 12. $y-y^{\prime}$ phase-space projections measured at the FRIB front end during a quadrupole scan. $V_{Q 3}$ denotes the voltage at the last quadrupole upstream of the Allison scanner.

\section{B. Quadrupole scan measurements}

A set of six measurements were made on a $50 \mu \mathrm{A}$ (measured separately by Faraday cup) $\mathrm{Ar}^{9+}$ ion beam using the $y$-plane Allison scanner, where $V_{Q 3}$, the voltage at the last quadrupole in the triplet upstream, varied from $0 \mathrm{~V}$ to $5000 \mathrm{~V}$ at $1000 \mathrm{~V}$ intervals.

Each scan was performed with $y$-spatial steps of $1 \mathrm{~mm}$ and voltage steps of $20 \mathrm{~V}$. Raw data were processed using the noise removal procedures outlined in Appendix D. The resulting data set was analyzed using both the conventional and improved methods as outlined in Sec. II. Three $y-y^{\prime}$ phase-space projections (with corrections) at different $V_{Q 3}$ are shown in Fig. 12. 
TABLE VI. Beam parameters of corresponding to the phase space distribution at $V_{Q 3}=1000 \mathrm{~V}$ in Fig. 12, computed with and without correction terms.

\begin{tabular}{lccc}
\hline \hline Parameters & $\begin{array}{c}\text { Conventional } \\
\text { analysis }\end{array}$ & $\begin{array}{c}\text { Phase space } \\
\text { correction }\end{array}$ & $\begin{array}{c}\text { Percentage } \\
\text { difference }\end{array}$ \\
\hline$\langle y\rangle[\mathrm{mm}]$ & 7.02 & 7.09 & $1.0 \%$ \\
$\left\langle y^{\prime}\right\rangle[\mathrm{mrad}]$ & 4.36 & 4.64 & $6.0 \%$ \\
$\langle y y\rangle\left[\mathrm{mm}^{2}\right]$ & 8.84 & 9.76 & $9.4 \%$ \\
$\left\langle y y^{\prime}\right\rangle\left[\mathrm{mm} \mathrm{mrad}^{\prime}\right]$ & 17.8 & 19.6 & $9.2 \%$ \\
$\left\langle y^{\prime} y^{\prime}\right\rangle\left[\mathrm{mrad}^{2}\right]$ & 76.9 & 80.8 & $4.8 \%$ \\
$\varepsilon_{\text {rms }}[\mathrm{mm} \mathrm{mrad}]$ & 19.0 & 20.1 & $5.5 \%$ \\
$I_{\text {total }}[\mu \mathrm{A}]$ & 36.8 & 39.6 & $7.1 \%$ \\
\hline \hline
\end{tabular}

To illustrate the differences between the conventional and improved analysis methods, we compute moments of the beam measured at $V_{Q 3}=1000 \mathrm{~V}$ both with and without geometric corrections. The calculations with geometric corrections use the post-Feb 2018 geometry in Table V, while the geometry's symmetric $\left(l=\left(l_{1}+l_{2}\right) / 2=\right.$ $4.962 \mathrm{~mm}$ on both ends) and thin-slit $(d=0 \mu \mathrm{m})$ counterpart gives results with both geometric features ignored.

Results are listed in Table VI. All second-order moments have centroids subtracted, i.e., $\left\langle y^{2}\right\rangle \equiv\left\langle(y-\langle y\rangle)^{2}\right\rangle$. We observe differences up to $\sim 10 \%$ in beam moments, thus confirming the importance of corrections for accurate measurements.

While the example above shows the magnitude of the corrections, it does not provide evidence on which result is more valid. If the corrections introduced in the analysis constitute an "improved" treatment over the conventional one, a set of quadrupole scan measurements analyzed with the former should exhibit better consistency than those analyzed with the conventional methods. This check is performed below.

Conclusive evidence of improvement arises from first order moments of the beam. Measurement results of a quadrupole scan and the linear transfer map at each setting can be used to form a system of linear equations:

$$
\left(\begin{array}{cc}
\mathbf{M}\left(V_{Q 3}=V_{1}\right) & \mathbf{I}_{2} \\
\vdots & \vdots \\
\mathbf{M}\left(V_{Q 3}=V_{n}\right) & \mathbf{I}_{2}
\end{array}\right)\left(\begin{array}{c}
\mathbf{x}_{\mathbf{i}} \\
\mathbf{x}_{\mathbf{0}}
\end{array}\right)=\left(\begin{array}{c}
\mathbf{x}_{\mathbf{f}}\left(V_{Q 3}=V_{1}\right) \\
\vdots \\
\mathbf{x}_{\mathbf{f}}\left(V_{Q 3}=V_{n}\right)
\end{array}\right) .
$$

Here, $V_{Q 3}$ is the voltage of the last quad, $\mathbf{I}_{\mathbf{2}}$ is the $2 \times 2$ identity matrix, $\mathbf{M}\left(V_{Q 3}\right)$ is the $2 \times 2$ transfer matrix consisting of the quadrupole at $V_{Q 3}$ and the quadrupoleto-scanner drift, and $\mathbf{x}=\left(\begin{array}{ll}x & x^{\prime}\end{array}\right)^{T}$ is the state vector with $\mathbf{x}_{\mathbf{i}}$ being the initial centroid position and angle entering the quad, $\mathbf{x}_{\mathbf{0}}$ being the position offset and tilting angle of the
TABLE VII. Solution of Eq. (11) for the quadrupole scan in Sec. IV B, with measurement results $x_{f}$ calculated using conventional and improved analysis method, respectively.

\begin{tabular}{lccccc}
\hline \hline & \multicolumn{2}{c}{ Beam } & & \multicolumn{2}{c}{ Scanner } \\
\cline { 2 - 3 } \cline { 6 - 6 } Analysis & $x_{i}[\mathrm{~mm}]$ & $x_{i}^{\prime}[\mathrm{mrad}]$ & & $x_{0}[\mathrm{~mm}]$ & $x_{0}^{\prime}[\mathrm{mrad}]$ \\
\hline Conventional & -5.9 & 37.7 & & -18.0 & -33.6 \\
Improved & -0.7 & 5.7 & & 3.0 & -1.2 \\
\hline \hline
\end{tabular}

Allison scanner with respect to the center line of the quad, and $\mathbf{x}_{\mathbf{f}}\left(V_{Q 3}\right)$ being the measured centroid position and angle corresponding to the measurement at $V_{Q 3}$.

Equation (11) solves for the initial centroid position and angle of the beam, as well as the position offset and tilting angle of the Allison scanner. $\mathbf{x}_{\mathbf{f}}\left(V_{Q 3}\right)$ is computed differently depending on whether the conventional or improved analysis is applied. Results listed in Table VII show that solutions from the improved analysis are much more realistic. Tilting angles of $33.6 \mathrm{mrad}$ would not go undetected in mechanical alignment, nor would a beam with centroid angles $\left\langle x^{\prime}\right\rangle=$ $37.7 \mathrm{mrad}$ transport through the downstream beam line without significant loss. Therefore, the experimental data support the validity of the improved analysis over the conventional treatment.

The large differences between the two sets of solutions in Table VII can be explained as follows. $\mathbf{x}_{\mathbf{f}}\left(V_{Q 3}\right)$ from the conventional analysis can be viewed as a perturbation to $\mathbf{x}_{\mathbf{f}}\left(V_{Q 3}\right)$ from the improved analysis. The sensitivity of the solutions $\mathbf{x}_{\mathbf{i}}$ and $\mathbf{x}_{\mathbf{0}}$ to the perturbation can be quantified by the condition number [16] of the coefficient matrix in Eq. (11). Quadrupole scan parameters corresponding to larger condition numbers will be more sensitive to the perturbation. For the case presented in Table VII, the condition number is large. Choosing scan parameters corresponding to a well-conditioned coefficient matrix with a smaller condition number will be conducive to error minimization. Such issues have been explored in $[17,18]$. This topic is beyond the scope of this paper, and will be discussed by the authors in forthcoming work.

\section{CONCLUSION}

We incorporated two important geometric features for Allison scanners into an improved model that extends the conventional analysis. Both effects were modeled with analytic formulas that have been verified by particle simulations. Asymmetric E-dipole placement alters the voltageto-angle relation for the selected beam, whereas slit thickness $\gtrsim$ slit width introduces additional scraping and requires a data point weight correction that is quadratic in voltage. These effects increase with the angular extent of the beam distribution, and can significantly change the results of phase space measurements relative to conventional analysis 
methods. Applying these corrections to Allison scanner data at the FRIB front end led to $(\sim 10 \%)$ changes in beam moments; these corrections were crucial for obtaining consistent results in a quadrupole scan, which in turn provided experimental verification for the improved analysis methods. Detailed knowledge of the phase space area contributing to each data point also allows one to deconvolve overlapping measurements when the device resolution exceeds the spacing between data points. This was demonstrated using synthetic data sets.

PYTHON programs incorporating the improved analysis and noise removal for measurement data are made available [4]. They are readily applicable to any Allison scanner given its device geometry to implement accurate analytic models. Tools will be updated as continued improvements are made.

\section{ACKNOWLEDGMENTS}

The authors would like to thank our colleagues at FRIB: Y. Hao, S. Lidia, P. Ostroumov, A. Plastun, Y. Yamazaki, T. Yoshimoto, and Q. Zhao for many fruitful discussions on the analysis scheme; $\mathrm{Z}$. Tong for his incisive advice on computing; S. Cogan, D. Omitto, and R. Shane for technical support on the Allison scanner measurements at FRIB; G. Machicoane, S. Renteria, and J. Stetson for their meticulous control over the ECR ion source at FRIB that made stable measurements possible. The authors are grateful to the anonymous referees who provided useful and detailed comments on the manuscript. Work supported by the U.S. DOE Office of Science under Cooperative Agreement DE-SC0000661 and the NSF under Grant No. PHY-1565546.

\section{APPENDIX A: NONPARAXIAL EFFECTS: WHEN THEY ARE NEGLIGIBLE}

This Appendix estimates the magnitude of nonparaxial effects in an Allison scanner and provides a simple condition to determine whether they are negligible.

Consider an idealized device geometry, i.e., symmetric E-dipole placement and thin slits, which corresponds to Case I in Fig. 2. Given $V_{0}$, a reference particle with the corresponding angle $x_{\text {ref }}^{\prime}$ reaches maximum $x$-displacement at the axial center $(z=l+L / 2)$. The ratio between the axial kinetic energy decrement $\Delta \mathcal{E} \equiv \mathcal{E}-\mathcal{E}_{\min }$ at this point and the original axial kinetic energy $\mathcal{E}$ of the ion measures the strength of nonparaxial effects.

Taking uniform hard-edge dipole fields and the paraxial approximation,

$$
x^{\prime \prime}(z)=\left\{\begin{array}{ll}
-\frac{q V_{0}}{g \mathcal{E}} & \text { if } l \leq z \leq L+l \\
0 & \text { otherwise }
\end{array} .\right.
$$

For $x(z=0)=0$ and $x^{\prime}(z=0)=x_{\text {ref }}^{\prime}=\frac{1}{2} \frac{q V_{0} L}{g \mathcal{E}}$, the particle position at the center of the device is

$$
\begin{aligned}
x(z=l+L / 2) & =\frac{1}{2} \frac{q V_{0}}{g \mathcal{E}} L\left(\frac{3 L}{8}+l\right) \\
& \approx \frac{1}{5} \frac{q V_{0}}{g \mathcal{E}} L^{2} .
\end{aligned}
$$

The energy change $\Delta \mathcal{E}$ is maximum at $z=l+L / 2$ where the particle has its closest approach to the E-dipole. The fractional energy change is

$$
\frac{\Delta \mathcal{E}}{\mathcal{E}}=\frac{1}{\mathcal{E}} \frac{x(z=l+L / 2)}{g / 2} q V_{0}=\frac{2}{5}\left(\frac{L}{g}\right)^{2}\left(\frac{q V_{0}}{\mathcal{E}}\right)^{2} .
$$

For nonparaxial effects to be negligible, such energy gain should be much smaller than the kinetic energy of the beam, i.e., $\Delta \mathcal{E} \ll \mathcal{E}$, or

$$
\frac{2}{5}\left(\frac{L}{g}\right)^{2}\left(\frac{q V_{0}}{\mathcal{E}}\right)^{2} \ll 1
$$

Taking the approximation $L / g \approx 10$ for a typical plate spacing, this condition reduces to

$$
40\left(\frac{Q}{A}\right)^{2}\left(\frac{e V_{0}}{\mathcal{E}_{0}[\mathrm{eV} / \mathrm{u}]}\right)^{2} \ll 1
$$

Here, for ions we take $\mathcal{E}_{0}=\mathcal{E} / A$ to be the kinetic energy per atomic mass unit and $q=Q e$ with $Q$ being the charge state and $e$ the elementary charge. Non-paraxial effects are negligible at $V_{0}$ values that satisfy the above condition. For example, consider the condition applied to the $\mathcal{E}_{0}=$ $12 \mathrm{keV} / \mathrm{u}^{40} \mathrm{Ar}^{9+}$ ion beam measured in the FRIB front end in Sec. IV. Even at $V_{0}=1000 \mathrm{~V}$, the condition reduces to $\approx 0.014 \ll 1$, which is well satisfied. Thus it is not surprising that the realistic and ballistic simulations agree well for typical parameters.

\section{APPENDIX B: REALISTIC MODEL}

A Python [19] code is employed to numerically integrate particle equations of motion in a realistic $x-z$ 2D field map of the device geometry that is generated from the electrostatic field code POISSON [20]. $E_{x}=-\partial \phi / \partial x$ and $E_{z}=\partial \phi / \partial z$ electric field data are exported from POISSON onto a high-resolution, uniform $x-z$ mesh $(d x=$ $d z=0.2 \mathrm{~mm}$ ), and then imported into the PYTHON code. Fields at the particle position are calculated using bilinear interpolation from the gridded field data [21].

In Fig. 13, the applied field potential $\phi$ of the FRIB Allison scanner is contoured showing enhanced detail near the entrance slit. Only half the geometry in the zoomed figure is contoured since $\phi(-x, z)=-\phi(x, z)$.

Particles are advanced with nonrelativistic equations of motion 

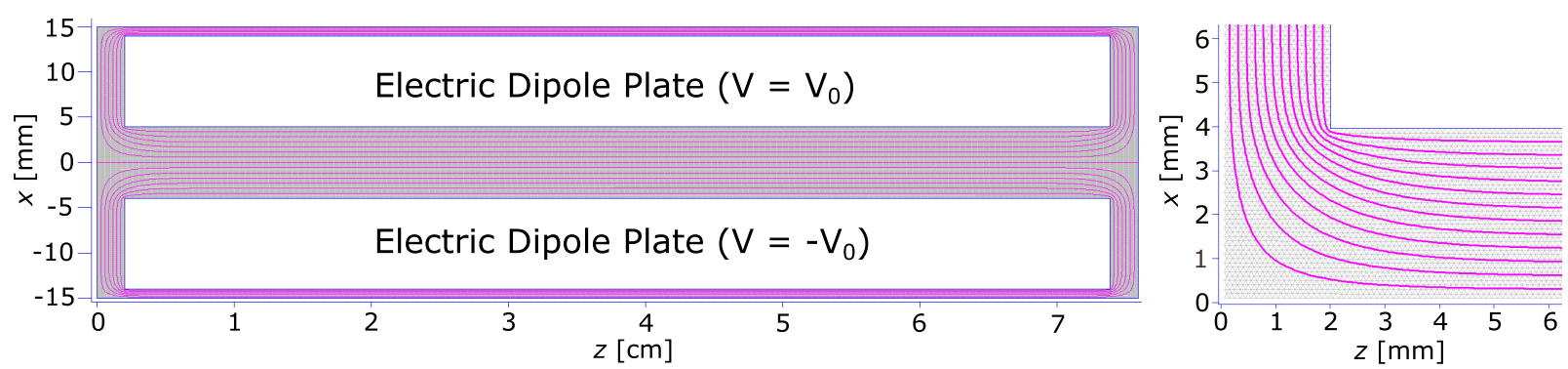

FIG. 13. Potential contours of the FRIB Allison scanner in Fig. 1, including details of the fringe structure in the vicinity of the entrance slit (right).

$$
\begin{aligned}
& \frac{d^{2} x}{d t^{2}}=\frac{q}{m} E_{x}, \\
& \frac{d^{2} z}{d t^{2}}=\frac{q}{m} E_{z} .
\end{aligned}
$$

The independent variable can be transformed exactly from time $t$ to axial coordinate $z$ giving

$$
\frac{d}{d z}\left[\begin{array}{c}
x \\
t \\
x^{\prime} \\
t^{\prime}
\end{array}\right]=\left[\begin{array}{c}
x^{\prime} \\
t^{\prime} \\
\left(\frac{q E_{x}}{m}-\frac{q E_{z}}{m} x^{\prime}\right) t^{\prime 2} \\
-\frac{q E_{z}}{m} t^{\prime 3}
\end{array}\right]
$$

where ${ }^{\prime} \equiv d / d z$, and $t^{\prime}=1 / v_{z}$.

This numerical model includes full fringe field effects entering and exiting the dipole field region, as well as nonparaxial effects due to energy change as the particle crosses potential lines. Image charges, beam space charge and scattering effects are neglected.

The state vector in Eq. (B1) describing the particle trajectory is advanced using the ODE package within Scientific Python (SCIPY) [22] for specified initial particle coordinate $x$, angle $x^{\prime}$, and dipole voltage $V_{0}$ (field data scaled). The code takes into account scraping on all boundaries. To solve for $x_{\max , \min }^{\prime}$, note that the corresponding trajectory must touch the slits at two points (see Fig. 2). We employ a numerical root-finding procedure to solve for the initial $x^{\prime}$ that connects the upstream point to the downstream point. $x_{\text {ref }}^{\prime}$ is solved analogously with the condition $x=0$ at both ends.

\section{APPENDIX C: ANALYTIC RESULTS}

Appendix C 1 sketches key steps in the derivation of the expressions in Table II using the geometric models shown in Fig. 2. Complications involved in Case IV are outlined in Appendix C 2.

\section{Sketch of derivation using geometric models}

Table II is mostly derived based on two simple rules: (1) trajectories can be shifted in $x$-position; and (2) changing the initial $x^{\prime}=x_{i}^{\prime}$ at $z=z_{i}$ by $\delta x^{\prime}$ results in a displacement $\delta x=\left(z-z_{i}\right) \delta x^{\prime}$ downstream. Subscripts $i$ and $f$ denote initial (entrance) and final (exit) locations, respectively.

In Fig. 14, these principles are applied to Case III (symmetric E-dipole placement, thick slits) to illustrate several calculations. We observe that among a uniform spatial distribution of particles entering the slit with $x_{\text {ref }}^{\prime}$, a fraction $s_{1} / s$ is collimated by the slit plate due to its thickness, where $s_{1}=x_{\text {ref }}^{\prime} d$. This explains the factor $\left(1-\frac{x_{\text {ref }}^{\prime} d}{s}\right)$ in the transmission coefficient $T\left(x_{\text {ref }}^{\prime}\right)$, which manifests itself in Fig. 3 as a horizontal shrinkage of the blue area in Case III in comparison with Case I.

To calculate $x_{\max }^{\prime}$, we note that the corresponding trajectory touches the slit plates at the positions shown in Fig. 14. Consider a particle with the same $x_{i}$ entering the slits with angle $x_{\text {ref. }}^{\prime}$. Applying the second rule, we obtain $s_{2}=\left(x_{\max }^{\prime}-x_{\text {ref }}^{\prime}\right)(d+2 l+L)$, while $s_{1}=x_{\text {ref }}^{\prime} d$ as calculated above. Therefore,

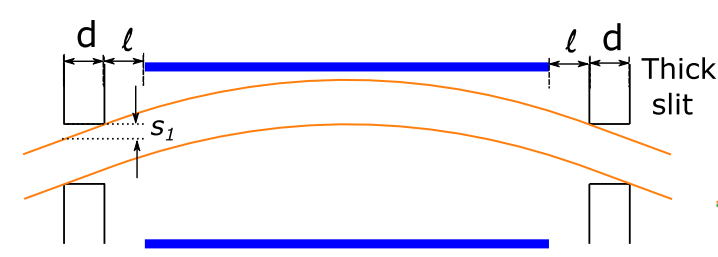

(a)

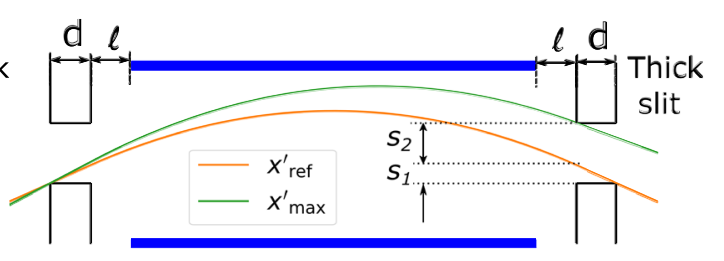

(b)

FIG. 14. How (a) shifting a trajectory for fixed angle and (b) changing the angle for fixed initial position allow one to calculate quantities listed in Table II. 


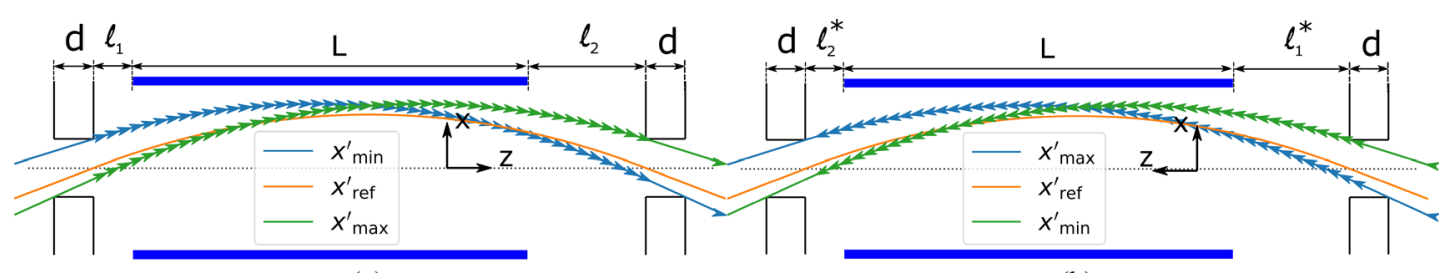

(a)

(b)

FIG. 15. Reversing particle velocity allows one to check results when the E-dipole placement is asymmetric.

$$
\begin{aligned}
s_{2} & =s-s_{1}, \\
\left(x_{\max }^{\prime}-x_{\mathrm{ref}}^{\prime}\right)(d+2 l+L) & =s-x_{\mathrm{ref}}^{\prime} d, \\
x_{\max }^{\prime}-x_{\mathrm{ref}}^{\prime} & =\frac{s-x_{\mathrm{ref}}^{\prime} d}{d+2 l+L},
\end{aligned}
$$

which is one of the results in Table II.

Note that the derivation above assumes bending within the E-dipole, which does not hold when $V_{0}=0$. In that case, instead of $\Delta x^{\prime}=2 s /(L+2 l+d)$, one can draw straight trajectories between furthest corners of the slits to see that $\Delta x^{\prime}=2 s /(L+2 l+2 d)$. Since $d \ll L+2 l$, the difference is very small and will be neglected.

The calculations for asymmetric E-dipole placement were carried out using the same principles, but one must note that $x_{i}^{\prime} \neq-x_{f}^{\prime}$ due to the asymmetry. This makes calculations in Case IV (asymmetric E-dipole placement, thick plate) much more complicated due to different scraping factors on the two ends. Rather than showing the tedious calculations, it is interesting to observe a succinct way to check the validity of the expressions in Case IV of Table II via direction reversal.

Figure 15(a) shows Case IV trajectories and Fig. 15(b) their direction-reversed counterparts, where the trajectories corresponding to maximum and minimum angles are interchanged depending on the direction of incoming particles. For the $x^{\prime}=x_{\max }^{\prime}$ trajectory in the normal case, the final particle angle equals $x_{\max }^{\prime}-\frac{q V_{0} L}{g \mathcal{E}}$, which equals $-x_{\min }^{\prime}$ for the direction-reversed case. Therefore, we can check whether

$$
\begin{aligned}
& \frac{1}{2} \frac{q V_{0} L}{g \mathcal{E}}\left(\frac{L+2 l_{2}}{\hat{L}}\right)+\frac{s-x_{\mathrm{ref}}^{\prime} d}{\hat{L}+d}-\frac{q V_{0} L}{g \mathcal{E}} \\
& =-\left[\frac{1}{2} \frac{q V_{0} L}{g \mathcal{E}}\left(\frac{L+2 l_{2}^{*}}{\hat{L}}\right)-\frac{1}{\hat{L}+d}\left(s-\frac{L+2 l_{1}^{*}}{L+2 l_{2}^{*}} x_{\mathrm{ref}}^{\prime} d\right)\right]
\end{aligned}
$$

where interslit distance $\hat{L}=L+l_{1}+l_{2}=L+l_{1}^{*}+l_{2}^{*}$ remains constant and $l_{1}^{*}=l_{2}, l_{2}^{*}=l_{1}$. All results for which the E-dipole placement is asymmetric have been verified with these procedures.

\section{Results for Case IV}

Procedures sketched above are applied to Case IV to obtain, for $l_{2}>l_{1}$ :

$$
\begin{aligned}
T & = \begin{cases}\frac{x_{\max }^{\prime}-x^{\prime}}{x_{\max }^{\prime}-x_{\mathrm{ref}}^{\prime}} c_{1} & \text { for } x_{\mathrm{ref}}^{\prime} \leq x^{\prime} \\
\frac{x_{\mathrm{ref}}^{\prime}-x^{\prime}}{x_{\mathrm{ref}}^{\prime}-\tilde{x}^{\prime}} c_{2}+\frac{x^{\prime}-\tilde{x}}{x_{\mathrm{ref}}^{\prime}-\tilde{x}^{\prime}} c_{1} & \text { for } \tilde{x}^{\prime} \leq x^{\prime}<x_{\mathrm{ref}}^{\prime}, \\
\frac{\tilde{x}^{\prime}-x^{\prime}}{\tilde{x}^{\prime}-x_{\min }^{\prime}} c_{2} & \text { for } x^{\prime}<\tilde{x}^{\prime}\end{cases} \\
W & =D_{1} \frac{L+l_{1}+l_{2}}{2 s^{2}},
\end{aligned}
$$

where

$$
\begin{aligned}
\tilde{x}^{\prime} & =x_{\mathrm{ref}}^{\prime}\left[1-\frac{2\left(l_{2}-l_{1}\right) d}{\left(L+l_{1}+l_{2}+2 d\right)\left(L+2 l_{2}\right)}\right], \\
c_{1} & =\left(1-\frac{x_{\mathrm{ref}}^{\prime} d}{s}\right), \\
c_{2} & =\left(1-\frac{\tilde{x}^{\prime} d}{s}\right), \\
D_{1} & =\frac{\left(c_{1} s\right)^{2}+\left(c_{2} s\right)^{2}}{2\left(L+l_{1}+l_{2}+d\right)}+\frac{s}{2}\left(c_{1}+c_{2}\right)\left(x_{\mathrm{ref}}^{\prime}-\tilde{x}^{\prime}\right) .
\end{aligned}
$$

Observe that $\tilde{x}^{\prime} \neq x_{\text {ref }}^{\prime}$ only if $l_{1} \neq l_{2}$ and $d \neq 0$, so the case $\tilde{x}^{\prime} \leq x^{\prime}<x_{\text {ref }}^{\prime}$ is only relevant when the E-dipole placement is asymmetric and slits are thick.

The reason there exists a third region in $T\left(x^{\prime}\right)$ is associated with a subtlety in the definition of $x_{\text {ref }}^{\prime}$. Previously, $x_{\text {ref }}^{\prime}$ is defined as the angle at which particles enter and exit the slits at the same $x$-position, with no distinction on which side of the slits we mean when the slits are thick.

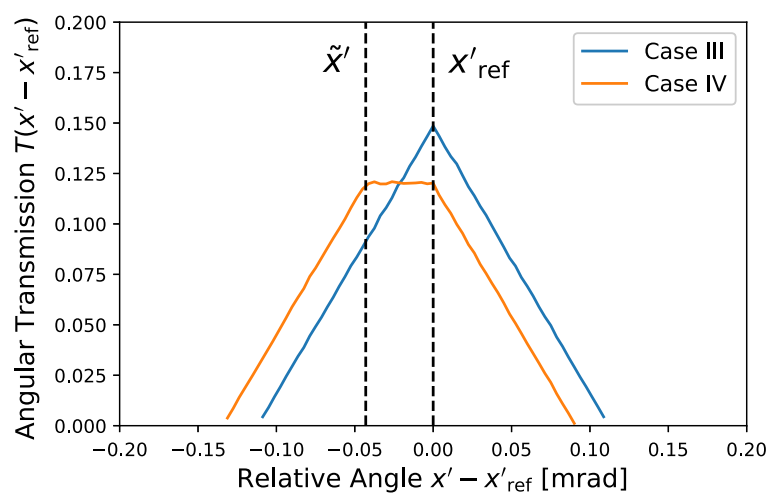

FIG. 16. Comparison between transmission ratio in Case III and IV when weight correction effect is large. 
Taking the inner facing side of the entrance slit as $z=0$, $x_{\text {ref }}^{\prime}$ is defined as the angle at which particles attain the same position at $z=0$ and $z=\hat{L}$ where $\hat{L}=L+l_{1}+l_{2}$ is the inter-slit distance. Then there is another angle $\tilde{x}^{\prime}$ with which the particles attain the same position at $z=-d$ and $z=\hat{L}+d \cdot x_{\text {ref }}^{\prime}=\tilde{x}^{\prime}$ in Case III because the entering and exiting angles are the same. However, in Case IV, since the entering and exiting angles are different due to the asymmetry, $x_{\text {ref }}^{\prime} \neq \tilde{x}^{\prime}$. When the $d / s$ ratio is large, the effects can be significant as shown in Fig. 16 for $d / s \simeq 4$.

\section{APPENDIX D: NOISE REMOVAL SCHEME}

The noise removal scheme for raw data is illustrated using an example measurement from the FRIB front end (see Sec. IV). The scheme first defines the beam region and uses the data points outside the beam region to characterize the background. Then the background is subtracted and unrealistic islands in the distribution are filtered. Details of the procedure are discussed below. Such noise thresholding is crucial for heavy ion beam measurements because the total beam current is typically $\sim 50 \mu \mathrm{A}$; this leaves many data points with merely $\sim \mathrm{nA}$ of collected currents which may only be $\sim 10$ times larger than noise fluctuations. Algorithms implementing the noise removal scheme applied in this study are incorporated in the PYTHON programs available at [4].

The noise removal scheme employed here has no capability to correct for ghost signals caused by particles that scatter after impacting the E-dipole plates; such ghost signals have been mitigated by using E-dipole plates whose surfaces have a staircase profile to reduce grazing incidences $[2,23]$.

\section{Specifying the beam region}

Figure 17 shows an ellipse that surrounds the entire beam distribution, thereby defining a region that contains the beam. Such a region can be designated by user input or generated automatically. It should be self-consistent in the sense that, after background subtraction and island filter, no nonzero data point touches the boundary specifying the beam region.

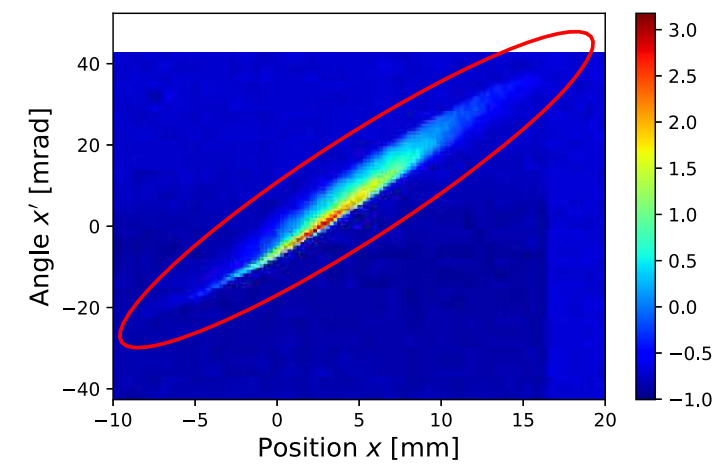

FIG. 17. Beam region is defined by the red ellipse.

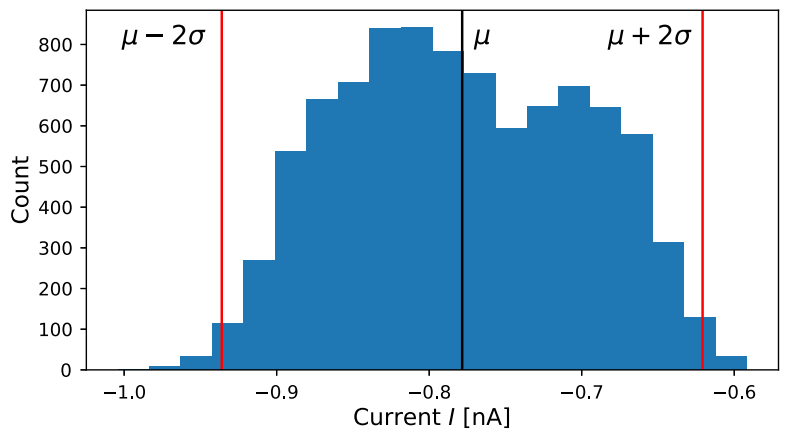

FIG. 18. Histogram of current values at data points outside the defined beam region.

\section{Background subtraction}

After designating the beam region, all exterior data points are used to characterize the background. In this example, the background noise has an average $\mu=-0.778 \mathrm{nA}$ with standard deviation $\sigma=0.079 \mathrm{nA}$. Figure 18 shows a histogram of the measured currents in exterior data points, where almost all values lie below $\mu+2 \sigma$. Therefore, a $\mu+2 \sigma$ cutoff is typically applied for interior data points, whereby any point with current $<\mu+2 \sigma$ is regarded as pure noise and assigned the value 0 . Subsequently, all remaining nonzero data points have $\mu$ subtracted from them to correct for the background. Note that $\mu<0$ in this case, which is likely due to amplifier characteristics. Figure 19 shows all nonzero data points after applying background subtraction to the raw data in Fig. 17.

\section{Island filter}

The beam distribution after background subtraction often contains spurious islands that probably arise from noise that exceeds the cutoff value. Figure 20 zooms in on the upper right sector of the beam distribution in Fig. 19 to show a number of islands before and after they are filtered.

The island filter we employ is a modification of the median filter widely used in speech and image processing [24]. For each nonzero data point, the island filter examines neighbors in an $n \times n$ grid and counts the number of nonzero points. If the number $<k$, the data point in question is assigned a zero value after the filter has processed all data points.

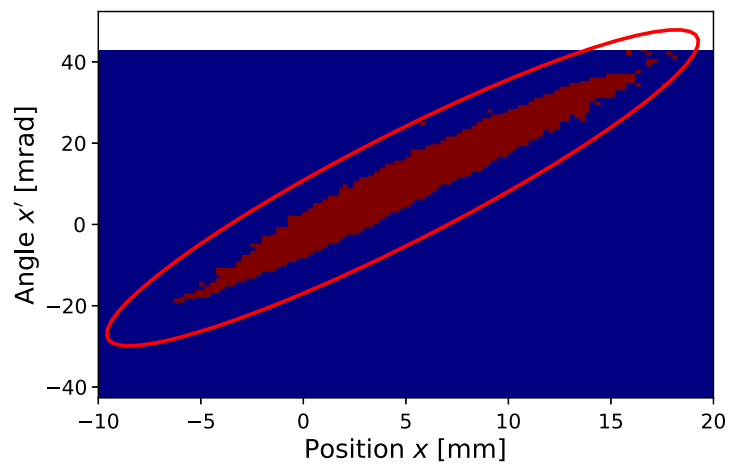

FIG. 19. Nonzero data points after background subtraction. 


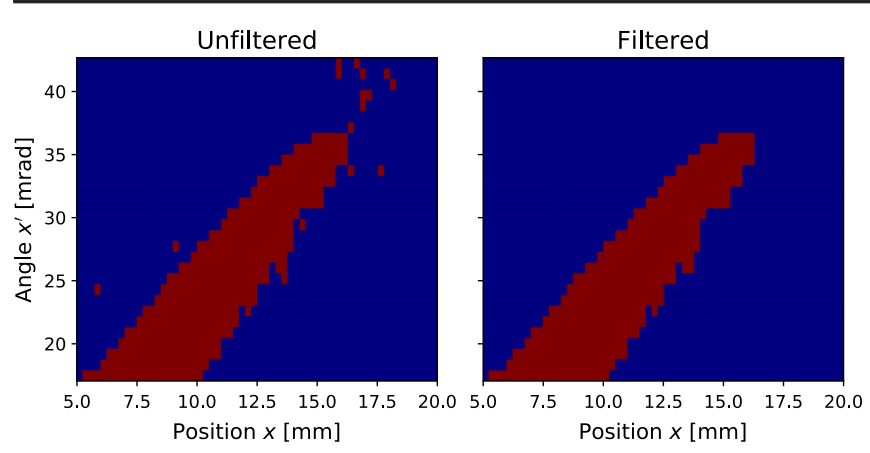

FIG. 20. Nonzero data points of the beam distribution, before and after applying the island filter.

The filter can be applied until it no longer has any effect on the distribution, which may require several iterations to enable layer-by-layer removal of clusters of islands.

In Fig. 20, $n=5$ and $k=3$, which means that if fewer than 3 data points amongst 24 neighboring points are nonzero, the data point at center is deemed part of a noise island and assigned the value zero. The filter takes two iterations to reach convergence in this case.

[1] P. W. Allison, J. D. Sherman, and D. B. Holtkamp, An emittance scanner for intense low-energy ion beams, IEEE Trans. Nucl. Sci. 30, 2204 (1983).

[2] M. Stockli, R. Welton, R. Keller, and M. Leitner, Emittance studies with an Allison scanner, Rev. Sci. Instrum. 77, 03B706 (2006).

[3] C. Y.J. Wong et al., in Proceedings, 6th International Beam Instrumentation Conference (IBIC2017), Grand Rapids, USA, 2017 (JACOW, Geneva, Switzerland, 2018), pp. $72-76$.

[4] https://github.com/AllisonScanner/Analysis.

[5] S. Lidia et al., in Proceedings, 4th International Beam Instrumentation Conference (IBIC2015), Melbourne, Australia, 2015 (JACOW, Geneva, Switzerland, 2016), pp. 226-230.

[6] M. Stockli, W. Blokland, T. Gorlov, B. Han, C. Long, T. Pennisi, and S. Assadi, in Proceedings of the 23rd Particle Accelerator Conference, Vancouver, Canada, 2009 (IEEE, Piscataway, NJ, 2009), pp. 3974-3976.

[7] R. D’Arcy, M. Alvarez, J. Gaynier, L. Prost, V. Scarpine, and A. Shemyakin, Characterisation of the PXIE Allison-type emittance scanner, Nucl. Instrum. Methods Phys. Res., Sect. A 815, 7 (2016).
[8] C. Ullmann, R. Berezov, J. FIls, R. Hollinger, V. Ivanova, O. Kester, W. Vinzenz, N. Chauvin, and O. Delferrire, in Proceedings, 5th International Particle Accelerator Conference (IPAC 2014), Dresden, Germany, 2014 (JACOW, Geneva, Switzerland, 2014), pp. 1120-1122.

[9] A. Laxdal, F. Ames, R. Baartman, W. Rawnsley, A. Sen, V. Verzilov, G. Waters, R. Hariwal, M. Kownacki, and R. Paris, in Proceedings, 27th Linear Accelerator Conference (LINAC2014), Geneva, Switzerland, 2014 (JACOW, Geneva, Switzerland, 2014), pp. 829-833.

[10] See Appendix C 2 for a subtle distinction in Case IV.

[11] M. Reiser, Theory and Design of Charged Particle Beams (John Wiley \& Sons, New York, 2008).

[12] S. M. Lund, T. Kikuchi, and R. C. Davidson, Generation of initial kinetic distributions for simulation of long-pulse charged particle beams with high space-charge intensity, Phys. Rev. ST Accel. Beams 12, 114801 (2009).

[13] https://git-scm.com/.

[14] J. Wei et al., in Proceedings, 28th International Linear Accelerator Conference (LINAC16), East Lansing, USA, September 25-30, 2016 (JACOW, Geneva, Switzerland, 2017), pp. 1-6.

[15] G. Machicoane, D. Cole, J. Ottarson, J. Stetson, and P. Zavodszky, ARTEMIS-B: A room-temperature test electron cyclotron resonance ion source for the National Superconducting Cyclotron Laboratory at Michigan State University, Rev. Sci. Instrum. 77, 03A322 (2006).

[16] J. W. Demmel, Applied Numerical Linear Algebra (Siam, Philadelphia, 1997).

[17] E. Prat and M. Aiba, Four-dimensional transverse beam matrix measurement using the multiple-quadrupole scan technique, Phys. Rev. ST Accel. Beams 17, 052801 (2014).

[18] C. Xiao, M. Maier, X. N. Du, P. Gerhard, L. Groening, S. Mickat, and H. Vormann, Rotating system for fourdimensional transverse rms-emittance measurements, Phys. Rev. Accel. Beams 19, 072802 (2016).

[19] https://www.python.org/.

[20] M. Menzel and H. K. Stokes, Users guide for the POISSON/SUPERFISH group of codes, Los Alamos National Lab, Technical Report No. LA-UR-87-115, 1987.

[21] C. K. Birdsall and A. B. Langdon, Plasma Physics via Computer Simulation (Taylor \& Francis, Boca Raton, 2004).

[22] https://www.scipy.org/.

[23] M. Stockli, M. Leitner, D. Moehs, R. Keller, and R. Welton, Beam dumping ghost signals in electric sweep scanners, AIP Conf. Proc. 763, 145 (2005).

[24] J. W. Tukey, Exploratory data analysis (Pearson, Boston, 1977). 